\title{
Well-posedness for nonlinear SPDEs with strongly continuous perturbation
}

\author{
Guy Vallet \\ LMAP UMR CNRS 5142, IPRA BP 1155, 64013 Pau Cedex, France \\ (guy.vallet@univ-pau.fr)
}

\author{
Aleksandra Zimmermann \\ Faculty of Mathematics, Thea-Leymann-Str. 9, 45127 Essen, Germany \\ (aleksandra.zimmermann@uni-due.de)
}

(MS Received 16 May 2019; Accepted 3 February 2020)

\begin{abstract}
We consider the well-posedness of a stochastic evolution problem in a bounded Lipschitz domain $D \subset \mathbb{R}^{d}$ with homogeneous Dirichlet boundary conditions and an initial condition in $L^{2}(D)$. The main technical difficulties in proving the result of existence and uniqueness of a solution arise from the nonlinear diffusion-convection operator in divergence form which is given by the sum of a Carathéodory function satisfying $p$-type growth associated with coercivity assumptions and a Lipschitz continuous perturbation. In particular, we consider the case $1<p<2$ with an appropriate lower bound on $p$ determined by the space dimension. Another difficulty arises from the fact that the additive stochastic perturbation with values in $L^{2}(D)$ on the right-hand side of the equation does not inherit the Sobolev spatial regularity from the solution as in the multiplicative noise case.
\end{abstract}

Keywords: Nonlinear SPDE; strongly continuous perturbation; additive stochastic forcing; pseudomonotone SPDE

2010 Mathematics subject classification: 60H15; 35K92; 35K55

\section{Introduction}

\subsection{Statement of the problem}

Let $(\Omega, \mathcal{F}, P)$ be a complete, countably generated probability space (e.g. the classical Wiener space), $D \subset \mathbb{R}^{d}$ be a bounded Lipschitz domain, $T>0, Q_{T}:=$ $(0, T) \times D$. We are interested in a result of existence and uniqueness of the solution to

$$
\begin{aligned}
\mathrm{d} u-\operatorname{div}(a(x, u, \nabla u)+F(u)) \mathrm{d} t & =\Phi \mathrm{d} W(t) & & \text { in } \Omega \times Q_{T}, \\
u & =0 & & \text { on } \Omega \times(0, T) \times \partial, \\
u(0, \cdot) & =u_{0} & & \in L^{2}(D) .
\end{aligned}
$$

The nonlinear diffusion-convection operator of Leray-Lions' type is defined as the sum of a Carathéodory function $a: D \times \mathbb{R}^{d+1} \rightarrow \mathbb{R}^{d}$ satisfying appropriate growth and coercivity assumptions which will be given below and $F: \mathbb{R} \rightarrow \mathbb{R}^{d}$ Lipschitz continuous with Lipschitz constant $L>0$, such that $F(0)=0$. On the right-hand side,

(C) The Author(s), 2020. Published by Cambridge University Press. This is an Open Access article, distributed under the terms of the Creative Commons Attribution licence (http:// creativecommons.org/licenses/by/4.0/), which permits unrestricted re-use, distribution, and reproduction in any medium, provided the original work is properly cited. 
$\Phi \in L^{2}\left(\Omega ; \mathcal{C}\left([0, T] ; H S\left(L^{2}(D)\right)\right)\right.$ is progressively measurable, where $H S\left(L^{2}(D)\right)$ is the space of Hilbert-Schmidt operators from $L^{2}(D)$ to $L^{2}(D)$. $W$ is a cylindrical Wiener process in $L^{2}(D)$ with respect to a filtration $\left(\mathcal{F}_{t}\right)_{t \in[0, T]}$, denoted $\left(\mathcal{F}_{t}\right)$ in the sequel, satisfying the usual assumptions. More precisely, $W$ is defined in the following sense (see, e.g. [3]): for a fixed orthonormal basis $\left(e_{n}\right)_{n \in \mathbb{N}^{*}}$ of $L^{2}(D)$ and a sequence of independent, real-valued $\left(\mathcal{F}_{t}\right)$-Brownian motions $\left(\beta_{n}\right)_{n \in \mathbb{N}^{*}}$, we define for any $t \in[0, T]$ and $u \in L^{2}(D),\langle W(t), u\rangle:=\sum_{n=1}^{\infty}\left(e_{n}, u\right)_{L^{2}(D)} \beta_{n}(t)$. One can check (see, e.g. [3, p. 85]) that for any $u \in L^{2}(D),\langle W, u\rangle$ is a real-valued, $\left(\mathcal{F}_{t}\right)$-Wiener process such that $\mathbb{E}[\langle W(t), u\rangle\langle W(s), v\rangle]=\min (t, s)(u, v)_{L^{2}(D)}$ for any $u, v \in L^{2}(D)$, for all $s, t \in[0, T]$. $W$ can be represented in the following way: for any sequence $\left(a_{n}\right) \in l^{1}\left(\mathbb{R}^{+}\right)$it is easy to check that $W(t):=\sum_{n=1}^{\infty} \sqrt{a_{n}}\left(e_{n} / \sqrt{a_{n}}\right) \beta_{n}(t)$ is a $Q$-Wiener process with positive definite, symmetric and nuclear covariance operator $Q=\operatorname{diag}\left(a_{n}\right)$ in the (bigger) Hilbert space

$$
U:=Q^{-1 / 2}\left(L^{2}(D)\right)
$$

which is obtained as the completion of $L^{2}(D)$ with respect to the norm $\|\cdot\|_{U}$ induced by the scalar product $(u, v)_{U}:=\left(Q^{1 / 2} u, Q^{1 / 2} v\right)_{L^{2}(D)}$. However, the stochastic integral $\int_{0}^{t} \Phi \mathrm{d} W(s), t \in[0, T]$, can be defined independently of the representation of $W$ and the choice of $Q$ by $\Phi \mathrm{d} W(t):=\sum_{n=1}^{\infty} \Phi\left(e_{n}\right) \mathrm{d} \beta_{n}(t)$.

\subsection{Motivation and former results}

The technical novelties of this contribution arise from the stochastic forcing of the nonlinear diffusion-convection operator $u \mapsto-\operatorname{div}(a(x, u, \nabla u)+F(u))$. The diffusion part $u \mapsto-\operatorname{div} a(x, u, \nabla u)$ is a monotone operator with $p$-growth and coercivity conditions for $2 d /(d+1)<p<\infty$ where $d \in \mathbb{N}^{*}$ is the space dimension (see $\S 1.3$ for more details). In particular, the assumptions include a class of $p$-Laplacian operators with $1<p<2, p$ appropriately bounded away from 1 . In contrast, the convection part $u \mapsto-\operatorname{div} F(u)$ is, in general, not monotone but strongly continuous. It is well-known that a semigroup representation of the solution in the sense of $[\mathbf{3}]$ is not available in this nonlinear case. Moreover, classical well-posedness theory for monotone SPDEs (see, e.g. [11]) and for locally monotone SPDEs (see [10]) does not apply for a general perturbation of the type - div $F(u)$ for Lipschitz continuous $F: \mathbb{R} \rightarrow \mathbb{R}^{d}$. Therefore, our aim is to show well-posedness results by using a semi-implicit Euler-Maruyama time discretization (i.e. implicit in the operator part and explicit in the noise one). From the a priori estimates, we get weak convergences of approximate solutions with respect to $(\omega, t, x)$, but compactness arguments can be applied uniquely with respect to the variables $(t, x)$. It is therefore not possible to identify the limit of the convection part of the operator. However, it is possible to show the existence of a martingale solution by adapting argumentations based on convergence in law and Skorokhod's representation theorem. Then, since a pathwise uniqueness result can be obtained by using a $L^{1}$ contraction principle, the existence of a strong solution follows by the argument of convergence in probability of Gyöngy and Krylov [8]. Those techniques are wellknown for evolution problems with a multiplicative stochastic perturbation and have been applied in, e.g. $[\mathbf{4}, \mathbf{6}]$ and by many other authors in the last few decades. 
In our case, this method allows to pass to the limit in the nonlinear convection operator. Taking advantage of the additive character of the stochastic perturbation, we can recover the stochastic integral by using a result detailed by Debussche et al. in [4]. Since the stochastic integral takes values in $L^{2}(D)$, it does not inherit spatial Sobolev regularity of the solution. Therefore, the compactness arguments are more subtle than in the case of a multiplicative stochastic perturbation as considered in [17] for the operator $u \mapsto-\operatorname{div}\left(|\nabla u|^{p-2} \nabla u+F(u)\right)$ with $p>2$.

\subsection{Assumptions on the nonlinear operator}

The vector field $a: D \times \mathbb{R} \times \mathbb{R}^{d} \rightarrow \mathbb{R}^{d},(x, \lambda, \xi) \mapsto a(x, \lambda, \xi)$ is a Carathéodory function in the sense that the mapping $(\lambda, \xi) \mapsto a(x, \lambda, \xi)$ is continuous for almost every $x \in D$ and $x \mapsto a(\cdot, \lambda, \xi)$ is measurable for every $(\lambda, \xi) \in \mathbb{R} \times \mathbb{R}^{d}$. In the following, let $2 d /(d+1)<p<\infty$ and $p^{\prime}=p /(p-1)$. We impose the following conditions:

(A1) $a$ is monotone with respect to its last variable only,

$$
[a(x, \lambda, \xi)-a(x, \lambda, \eta)] \cdot(\xi-\eta) \geqslant 0
$$

for all $\lambda \in \mathbb{R}, \xi, \eta \in \mathbb{R}^{d}$ and almost every $x \in D$.

$(A 2)$ There exists $\kappa \in L^{1}(D)$, some constants $C_{a}^{1}>0, C_{a}^{2} \geqslant 0, C_{a}^{3} \geqslant 0$ and a nonnegative function $g \in L^{p^{\prime}}(D)$ such that

$$
\begin{gathered}
a(x, \lambda, \xi) \cdot \xi \geqslant \kappa(x)+C_{a}^{1}|\xi|^{p}, \\
|a(x, \lambda, \xi)| \leqslant C_{a}^{2}|\xi|^{p-1}+C_{a}^{3}|\lambda|^{p-1}+g(x)
\end{gathered}
$$

for all $\lambda \in \mathbb{R}, \xi \in \mathbb{R}^{d}$ and almost every $x \in D$.

(A3) There exists a constant $C_{a}^{4} \geqslant 0$ and a nonnegative function $h \in L^{p^{\prime}}(D)$ such that

$$
\left|a\left(x, \lambda_{1}, \xi\right)-a\left(x, \lambda_{2}, \xi\right)\right| \leqslant\left[C_{a}^{4}|\xi|^{p-1}+h(x)\right]\left|\lambda_{1}-\lambda_{2}\right|
$$

for all $\lambda_{1}, \lambda_{2} \in \mathbb{R}$, for all $\xi \in \mathbb{R}^{d}$ and almost all $x \in D$.

From assumptions $(A 1)-(A 2)$ it follows that the nonlinear operator

$$
\mathcal{A}: W_{0}^{1, p}(D) \rightarrow W^{-1, p^{\prime}}(D), \quad u \mapsto \mathcal{A}(u):=-\operatorname{div} a(x, u, \nabla u)
$$

is well-defined, hemicontinuous and pseudomonotone (see [12, lemma 2.32]). From assumption (1.3) of (A2), it follows that $\mathcal{A}$ is coercive.

Since $p>2 d /(d+1)>2 d /(d+2)$, this implies that $p^{*}:=d p /(d-p)>2$ when $p<d$ and $W_{0}^{1, p}(D)$ is compactly embedded into $L^{2}(D)$. In addition, the stricter condition $p>2 d /(d+1)$ implies that $p^{\prime}<p^{*}$. Therefore, $L^{p^{*}}(D)$ is continuously embedded in $L^{p^{\prime}}(D)$ and the operator $u \in W_{0}^{1, p}(D) \mapsto-\operatorname{div}(a(x, u, \nabla u)+F(u))$ is well defined. An example of an operator satisfying $(A 1)-(A 3)$ is given by $\mathcal{A}(u)=$ $-\operatorname{div}\left(\varphi(x, u)|\nabla u|^{p-2} \nabla u\right)$ for a strictly positive, Lipschitz continuous (with respect to the second variable) and bounded function $\varphi$. 


\subsection{Strong solutions}

Let us recall the notion of strong solution to (1.1):

Definition 1.1. A (strong) solution to (1.1) is a predictable process $u \in$ $L^{2}\left(\Omega ; \mathcal{C}\left([0, T] ; L^{2}(D)\right)\right) \cap L^{p}\left(\Omega ; L^{p}\left(0, T ; W_{0}^{1, p}(D)\right)\right)$ such that $u(0, \cdot)=u_{0}$ in $L^{2}(D)$ and

$$
u(t)-u_{0}-\int_{0}^{t} \operatorname{div}(a(x, u, \nabla u)+F(u)) \mathrm{d} s=\int_{0}^{t} \Phi \mathrm{d} W(s),
$$

in $L^{2}(D)$ for all $t \in[0, T]$, a.s. in $\Omega$.

\subsection{Main result and outline}

TheOREM 1.1. For any $u_{0} \in L^{2}(D)$ and any progressively measurable $\Phi \in$ $L^{2}\left(\Omega ; \mathcal{C}\left([0, T] ; H S\left(L^{2}(D)\right)\right)\right.$ there exists a strong solution to (1.1).

The proof of theorem 1.1 is contained in the following section. It is based on an approximation procedure by a time discretization of (1.1) introduced in $\S 2.1$. Since there is a lack of compactness with respect to $\omega \in \Omega$, we use the theorems of Prokhorov and Skorokhod to obtain a.s. the convergence of the sequence of approximate solutions [2].

\section{Proof of theorem 1.1}

\subsection{Time discretization}

For $N \in \mathbb{N}^{*}$, let $0=t_{0}<t_{1}<\ldots<t_{N}=T$ be an equidistant subdivision of the interval $[0, T]$ with $\tau:=T / N=t_{k+1}-t_{k}$ for all $k=0, \cdots, N-1$. For $u_{0} \in L^{2}(D)$, $u^{0}=u_{0}^{\tau}$ given by lemma A.1 and $\Phi_{k}:=\Phi\left(t_{k}\right)$ for $k=0, \ldots, N-1$, we introduce the semi-implicit Euler-Maruyama scheme

$$
u^{k+1}-u^{k}-\tau \operatorname{div}\left(a\left(x, u^{k+1}, \nabla u^{k+1}\right)+F\left(u^{k+1}\right)\right)=\Phi_{k} \Delta_{k+1} W,
$$

where $\Phi_{k} \Delta_{k+1} W:=\Phi\left(t_{k}\right)\left(W\left(t_{k+1}\right)-W\left(t_{k}\right)\right)$ for $k=0, \ldots, N-1$.

Lemma 2.1. For any $\tau>0$ and any given $\mathcal{F}$-measurable $u^{k} \in L^{2}(D)$, there exists a unique $\mathcal{F}$-measurable function $u^{k+1}: \Omega \rightarrow W_{0}^{1, p}(D)$ satisfying $(2.1)$ in $L^{2}(D)$, for a.e. $\omega \in \Omega$. If moreover $u^{k}$ is $\mathcal{F}_{t_{k}}$-measurable, then $u^{k+1}$ is $\mathcal{F}_{t_{k+1}}$-measurable.

Proof. The operator $A_{\tau}: W_{0}^{1, p}(D) \rightarrow W^{-1, p^{\prime}}(D)$ defined by

$$
\left\langle A_{\tau}(u), v\right\rangle_{W^{-1, p^{\prime}}, W_{0}^{1, p}}:=(u, v)_{2}+\tau \int_{D}(a(x, u, \nabla u)+F(u)) \cdot \nabla v \mathrm{~d} x
$$

for $u, v \in W_{0}^{1, p}(D)$ is a pseudomonotone operator, since it is the sum of a pseudomonotone operator and a strongly continuous perturbation (see, e.g. [12, corollary 2.12, $\S 2.4 .3]$ ). Thanks to $(A 2)$, condition (1.3) and Gauss-Green's 
theorem, $A_{\tau}$ is coercive, i.e.

$$
\lim _{\|u\|_{W_{0}^{1, p}(D)} \rightarrow \infty} \frac{\left\langle A_{\tau}(u), u\right\rangle_{W^{-1, p^{\prime}}(D), W_{0}^{1, p}(D)}}{\|u\|_{W_{0}^{1, p}(D)}}=+\infty .
$$

Let $V \subset W_{0}^{1, p}(D)$ be bounded, i.e. $\sup _{u \in V}\|u\|_{W_{0}^{1, p}(D)} \leqslant C_{V}$ for some constant $C_{V} \geqslant 0$. In the following, $C_{i} \geqslant 0$, for $i=1,2,3 \ldots$ will be constants. For any $u \in V$ and any $v \in W_{0}^{1, p}(D)$ such that $\|v\|_{W_{0}^{1, p}(D)} \leqslant 1$ we have

$$
\left|\left\langle A_{\tau}(u), v\right\rangle_{W^{-1, p^{\prime}}, W_{0}^{1, p}}\right| \leqslant I_{1}+I_{2}+I_{3} .
$$

Using Cauchy-Schwarz inequality and the continuous embedding of $W_{0}^{1, p}(D)$ into $L^{2}(D)$ for $p \geqslant 2 d /(d+1)$, we have

$$
I_{1}=\left|(u, v)_{2}\right| \leqslant\|u\|_{2}\|v\|_{2} \leqslant C_{1}\|u\|_{W_{0}^{1, p}(D)}\|v\|_{W_{0}^{1, p}(D)} \leqslant C_{1} C_{V} .
$$

From (1.4) of (A2), Young's and Poincaré's inequalities it follows that

$$
\begin{aligned}
I_{2} & =\tau \int_{D}|a(x, u, \nabla u) \cdot \nabla v| \mathrm{d} x \\
& \leqslant \frac{\tau}{p^{\prime}} C_{2} \int_{D}|\nabla u|^{p}+|u|^{p}+g(x)^{p^{\prime}} \mathrm{d} x+\frac{\tau}{p}\|v\|_{W_{0}^{1, p}(D)}^{p} \\
& \leqslant \frac{\tau}{p^{\prime}} C_{2}\left(\|u\|_{W_{0}^{1, p}(D)}^{p}+\|g\|_{p^{\prime}}^{p^{\prime}}\right)+\frac{\tau}{p} \leqslant C_{3}\left(C_{V}^{p}+\|g\|_{p^{\prime}}^{p^{\prime}}+1\right) .
\end{aligned}
$$

We recall that thanks to the condition $p>2 d /(d+1)$, it follows that $L^{p^{*}}(D)$ is continuously embedded into $L^{p^{\prime}}(D)$ and therefore we can estimate

$$
\begin{aligned}
I_{3} & =\tau \int_{D}|F(u)||\nabla v| \mathrm{d} x \leqslant \frac{\tau}{p^{\prime}} \int_{D}|F(u)|^{p^{\prime}} \mathrm{d} x+\frac{\tau}{p}\|v\|_{W_{0}^{1, p}(D)}^{p} \\
& \leqslant \frac{\tau}{p^{\prime}} L^{p^{\prime}}\|u\|_{p^{\prime}}^{p^{\prime}}+\frac{\tau}{p} \leqslant C\|u\|_{p^{*}}^{p^{\prime}}+\frac{\tau}{p} \leqslant C_{4}\left(\|u\|_{W_{0}^{1, p}(D)}^{p}+1\right) \leqslant C_{4}\left(C_{V}^{p}+1\right),
\end{aligned}
$$

where $L>0$ is the Lipschitz constant of $F$. From equations (2.2)-(2.5) it follows that $A_{\tau}$ is bounded and, by Brezis' theorem (see, e.g. [12, theorem 2.6 on p. 33]), $A_{\tau}$ is onto $W^{-1, p^{\prime}}(D)$. In order to show that $A_{\tau}$ is injective, we fix $f \in W^{-1, p^{\prime}}(D)$ and assume that there exist $u_{1}, u_{2} \in W_{0}^{1, p}(D)$ such that $A_{\tau}\left(u_{1}\right)=A_{\tau}\left(u_{2}\right)=f$ in $W^{-1, p^{\prime}}(D)$. Then, for the non-decreasing Lipschitz continuous approximation of the sign function $\left(\operatorname{sign}_{\delta}\right)_{\delta \geqslant 0}$ defined for all $r \in \mathbb{R}$ by $\operatorname{sign}_{\delta}(r)=\max (-1, \min (1, r / \delta))$, the chain rule for Sobolev functions yields $J_{1}+J_{2}+J_{3}=0$, where

$$
\begin{aligned}
J_{1} & =\int_{D}\left(u_{1}-u_{2}\right) \operatorname{sign}_{\delta}\left(u_{1}-u_{2}\right) \mathrm{d} x \\
J_{2} & =\int_{D} \operatorname{sign}_{\delta}^{\prime}\left(u_{1}-u_{2}\right)\left(a\left(x, u_{1}, \nabla u_{1}\right)-a\left(x, u_{2}, \nabla u_{2}\right)\right) \cdot \nabla\left(u_{1}-u_{2}\right) \mathrm{d} x \\
J_{3} & =\int_{D} \operatorname{sign}_{\delta}^{\prime}\left(u_{1}-u_{2}\right)\left(F\left(u_{1}\right)-F\left(u_{2}\right)\right) \cdot \nabla\left(u_{1}-u_{2}\right) \mathrm{d} x
\end{aligned}
$$


It follows immediately that $\lim _{\delta \rightarrow 0^{+}} J_{1}=\int_{D}\left|u_{1}-u_{2}\right| \mathrm{d} x$. Now we write $J_{2}=J_{2,1}+$ $J_{2,2}$, where, by $(A 1)$,

$$
J_{2,1}=\int_{D} \operatorname{sign}_{\delta}^{\prime}\left(u_{1}-u_{2}\right)\left(a\left(x, u_{1}, \nabla u_{1}\right)-a\left(x, u_{1}, \nabla u_{2}\right)\right) \cdot \nabla\left(u_{1}-u_{2}\right) \mathrm{d} x \geqslant 0
$$

and by $(A 3)$

$$
\begin{aligned}
\left|J_{2,2}\right| & \leqslant \int_{D} \operatorname{sign}_{\delta}^{\prime}\left(u_{1}-u_{2}\right)\left|a\left(x, u_{1}, \nabla u_{2}\right)-a\left(x, u_{2}, \nabla u_{2}\right)\right|\left|\nabla\left(u_{1}-u_{2}\right)\right| \mathrm{d} x \\
& \leqslant \frac{1}{\delta} \int_{\left\{\left|u_{1}-u_{2}\right|<\delta\right\}}\left(C_{a}^{4}\left|\nabla u_{2}\right|^{p-1}+h(x)\right)\left|u_{1}-u_{2}\right|\left|\nabla\left(u_{1}-u_{2}\right)\right| \mathrm{d} x \\
& \leqslant C\left(\int_{D}\left|\nabla u_{2}\right|^{p}+h(x)^{p^{\prime}} \mathrm{d} x\right)^{1 / p^{\prime}}\left(\int_{\left\{\left|u_{1}-u_{2}\right|<\delta\right\}}\left|\nabla\left(u_{1}-u_{2}\right)\right|^{p} \mathrm{~d} x\right)^{1 / p} .
\end{aligned}
$$

Since $\nabla\left(u_{1}-u_{2}\right)=0$ a.e. in $\left\{u_{1}=u_{2}\right\}$ for Sobolev functions, $\lim _{\delta \rightarrow 0^{+}}\left|J_{2,2}\right|=0$ and $\lim \sup _{\delta \rightarrow 0^{+}} J_{2} \geqslant 0$. Since $F$ is Lipschitz continuous with Lipschitz constant $L>0$, we have

$$
\left|J_{3}\right| \leqslant \frac{1}{\delta} \int_{\left\{\left|u_{1}-u_{2}\right|<\delta\right\}} L\left|u_{1}-u_{2}\right|\left|\nabla\left(u_{1}-u_{2}\right)\right| \mathrm{d} x,
$$

thus $\lim _{\delta \rightarrow 0^{+}} J_{3}=0$. Combining the above results, we obtain $u_{1}=u_{2}$.

It is left to show that $A_{\tau}^{-1}: W^{-1, p^{\prime}}(D) \rightarrow W_{0}^{1, p}(D)$ is demi-continuous. For $f \in W^{-1, p^{\prime}}(D)$ and $u$ such that $A_{\tau}(u)=f$, using Gauss-Green's theorem on the convection term, we get $\langle f, u\rangle_{W^{-1, p^{\prime}}(D), W_{0}^{1, p}(D)}=\|u\|_{2}^{2}+\tau \int_{D} a(x, u, \nabla u) \cdot \nabla u \mathrm{~d} x$.

By $(A 2), \quad \int_{D} a(x, u, \nabla u) \cdot \nabla u \mathrm{~d} x \geqslant-\|\kappa\|_{1}+C_{a}^{1}\|\nabla u\|_{p}^{p}$ and therefore, using Young's inequality, for any $\delta>0$ we get

$$
\begin{aligned}
\|u\|_{2}^{2}-\tau\|\kappa\|_{1}+\tau C_{a}^{1}\|\nabla u\|_{p}^{p} & \leqslant\langle f, u\rangle_{W^{-1, p^{\prime}}(D), W_{0}^{1, p}(D)} \\
& \leqslant \frac{C_{\tau, \delta}}{p^{\prime}}\|f\|_{W^{-1, p^{\prime}}(D)}^{p^{\prime}}+\frac{\tau \delta}{p}\|\nabla u\|_{p}^{p}
\end{aligned}
$$

with a constant $C_{\tau, \delta} \geqslant 0$. Let $\left(f_{n}\right) \subset W^{-1, p^{\prime}}(D)$ be a sequence converging to $f$ in $W^{-1, p^{\prime}}(D)$. For all $n \in \mathbb{N}^{*}$, we define

$$
u_{n}:=A_{\tau}^{-1}\left(f_{n}\right)
$$

From (2.6) it follows that there exists a not relabelled subsequence of $\left(u_{n}\right), u \in$ $W_{0}^{1, p}(D)$ and $B$ in $L^{p^{\prime}}(D)^{d}$ such that $u_{n} \rightarrow u$ in $W_{0}^{1, p}(D), u_{n} \rightarrow u$ in $L^{2}(D)$ thanks to $p^{*}>2$ and $a\left(x, u_{n}, \nabla u_{n}\right) \rightarrow B$ in $L^{p^{\prime}}(D)^{d}$ for $n \rightarrow \infty$. Using these convergence 
results and (2.7), we get

$$
\begin{aligned}
& \|u\|_{2}^{2}+\tau \limsup _{n \rightarrow \infty} \int_{D} a\left(x, u_{n}, \nabla u_{n}\right) \cdot \nabla u_{n} \mathrm{~d} x \\
& \quad=\langle f, u\rangle_{W^{-1, p^{\prime}}(D), W_{0}^{1, p}(D)}=\|u\|_{2}^{2}+\tau \int_{D} B \cdot \nabla u \mathrm{~d} x .
\end{aligned}
$$

Thus, from (2.8) it follows that

$$
\begin{aligned}
& \limsup _{n \rightarrow \infty}\left\langle A_{\tau}\left(u_{n}\right), u_{n}-u\right\rangle_{W^{-1, p^{\prime}}(D), W_{0}^{1, p}(D)} \\
& \quad=\limsup _{n \rightarrow \infty} \int_{D} a\left(x, u_{n}, \nabla u_{n}\right) \cdot \nabla u_{n} \mathrm{~d} x-\int_{D} B \cdot \nabla u \mathrm{~d} x=0
\end{aligned}
$$

and since $A_{\tau}$ is pseudomonotone, (2.9) implies $A_{\tau} u=f$.

Consequently, $u_{n}=A_{\tau}^{-1}\left(f_{n}\right) \rightarrow A_{\tau}^{-1}(f)=u$ in $W_{0}^{1, p}(D)$, a priori for a subsequence. Since $u$ is unique, it follows that the whole sequence $\left(u_{n}\right)$ converges to $u$ weakly in $W_{0}^{1, p}(D)$ for $n \rightarrow \infty$ and $A_{\tau}^{-1}$ is demi-continuous.

By assumption, $\Phi_{k} \Delta_{k+1} W+u^{k}$ is $\mathcal{F}$ (resp. $\mathcal{F}_{t_{k+1}}$ ) measurable, thus

$$
u_{k+1}=A_{\tau}^{-1}\left(\Phi_{k} \Delta_{k+1} W+u^{k}\right)
$$

is weakly $\mathcal{F}$ (resp. $\mathcal{F}_{t_{k+1}}$ ) measurable, thus measurable by the theorem of Pettis (see $\left[\mathbf{1 8}, \mathrm{V} .4\right.$, p. 131]) since $W_{0}^{1, p}(D)$ is separable. The lemma is proved.

\subsection{Estimates}

Lemma 2.2. For $u_{0} \in L^{2}(D)$, and $k=0, \ldots, N-1$, let $u^{k+1}$ be the solution to (2.1). Then,

$$
\begin{aligned}
& \frac{1}{2} \mathbb{E}\left(\left\|u^{k+1}\right\|_{2}^{2}-\left\|u^{k}\right\|_{2}^{2}\right)+\frac{1}{4} \mathbb{E}\left\|u^{k+1}-u^{k}\right\|_{2}^{2}+\tau C_{a}^{1} \mathbb{E}\left\|\nabla u^{k+1}\right\|_{p}^{p} \\
& \quad \leqslant \tau\|\kappa\|_{1}+\tau \mathbb{E}\left\|\Phi_{k}\right\|_{H S\left(L^{2}(D)\right)}^{2} .
\end{aligned}
$$

Proof. Taking $u^{k+1}$ as a test function in (2.1), we get

$$
\begin{aligned}
& \left(u^{k+1}-u^{k}, u^{k+1}\right)_{2}-\tau\left\langle\operatorname{div}\left(a\left(x, u^{k+1}, \nabla u^{k+1}\right)+F\left(u^{k+1}\right)\right), u^{k+1}\right\rangle_{W^{-1, p^{\prime}}(D), W_{0}^{1, p}(D)} \\
& \quad=\left(\Phi_{k} \Delta_{k+1} W, u^{k+1}\right)_{2} \Leftrightarrow I_{1}+I_{2}+I_{3}=I_{4}
\end{aligned}
$$

where $I_{1}:=\left(u^{k+1}-u^{k}, u^{k+1}\right)_{2}=\frac{1}{2}\left(\left\|u^{k+1}\right\|_{2}^{2}-\left\|u^{k}\right\|_{2}^{2}+\left\|u^{k+1}-u^{k}\right\|_{2}^{2}\right)$, and, using $(A 2), I_{2}:=\tau \int_{D} a\left(x, u^{k+1}, \nabla u^{k+1}\right) \cdot \nabla u \mathrm{~d} x \geqslant-\tau\|\kappa\|_{1}+\tau C_{a}^{1}\|\nabla u\|_{p}^{p}$. 
From Gauss-Green's theorem it follows that

$$
I_{3}=\tau \int_{D} F\left(u^{k+1}\right) \cdot \nabla u^{k+1} \mathrm{~d} x=0
$$

and we still have to estimate $I_{4}=\left(\Phi_{k} \Delta_{k+1} W, u^{k+1}-u^{k}\right)_{2}+\left(\Phi_{k} \Delta_{k+1} W, u^{k}\right)_{2}$. Combining (2.11) with the above estimates and taking expectation we arrive at

$$
\begin{aligned}
& \frac{1}{2} \mathbb{E}\left(\left\|u^{k+1}\right\|_{2}^{2}-\left\|u^{k}\right\|_{2}^{2}+\left\|u^{k+1}-u^{k}\right\|_{2}^{2}\right)+\tau C_{a}^{1} \mathbb{E}\|\nabla u\|_{p}^{p} \\
& \quad \leqslant \tau\|\kappa\|_{1}+\mathbb{E}\left(\Phi_{k} \Delta_{k+1} W, u^{k+1}-u^{k}\right)_{2}+\mathbb{E}\left(\Phi_{k} \Delta_{k+1} W, u^{k}\right)_{2} .
\end{aligned}
$$

Since $u^{k}$ is $\mathcal{F}_{t_{k}}$-measurable and $W\left(t_{k+1}\right)-W\left(t_{k}\right)$ is $\mathcal{F}_{t_{k}}$-independent, we have

$$
\mathbb{E}\left(\Phi_{k} \Delta_{k+1} W, u^{k}\right)_{2}=\mathbb{E}\left(u^{k}, \mathbb{E}\left[\Phi_{k}\left(W\left(t_{k+1}\right)-W\left(t_{k}\right)\right) \mid \mathcal{F}_{t_{k}}\right]\right)_{2}=0 .
$$

Using Hölder's and Young's inequalities it follows that for any $\alpha>0$

$$
\mathbb{E}\left(\Phi_{k} \Delta_{k+1} W, u^{k+1}-u^{k}\right)_{2} \leqslant \frac{1}{2}\left(\frac{1}{\alpha} \mathbb{E}\left\|\int_{t_{k}}^{t_{k+1}} \Phi_{k} \mathrm{~d} W(t)\right\|_{2}^{2}+\alpha \mathbb{E}\left\|u^{k+1}-u^{k}\right\|_{2}^{2}\right) .
$$

By Itô's isometry, setting $\alpha=\frac{1}{2}$ in $(2.12)$ yields

$$
\begin{aligned}
& \mathbb{E}\left(\Phi_{k} \Delta_{k+1} W, u^{k+1}-u^{k}\right) \\
& \quad \leqslant \mathbb{E} \int_{t_{k}}^{t_{k+1}}\left\|\Phi_{k}\right\|_{H S\left(L^{2}(D)\right)}^{2} \mathrm{~d} t+\frac{1}{4} \mathbb{E}\left\|u^{k+1}-u^{k}\right\|_{2}^{2} \\
& \quad=\tau \mathbb{E}\left\|\Phi_{k}\right\|_{H S\left(L^{2}(D)\right)}^{2}+\frac{1}{4} \mathbb{E}\left\|u^{k+1}-u^{k}\right\|_{2}^{2},
\end{aligned}
$$

and therefore (2.10) holds.

Definition 2.1. For $N \in \mathbb{N}^{*}, \tau>0$, we introduce the right-continuous step function

$$
u_{N}^{r}(t)=\sum_{k=0}^{N-1} u^{k+1} \chi_{\left[t_{k}, t_{k+1}\right)}(t), \quad t \in[0, T),
$$

the left-continuous, $\left(\mathcal{F}_{t}\right)$-adapted step function

$$
\Phi_{N}(t)=\sum_{k=0}^{N-1} \Phi_{k} \chi_{\left(t_{k}, t_{k+1}\right]}(t), \quad t \in(0, T]
$$

the continuous, square-integrable $\left(\mathcal{F}_{t}\right)$-martingale

$$
M_{N}(t)=\int_{0}^{t} \Phi_{N} \mathrm{~d} W(s), \quad t \in[0, T]
$$


and the piecewise affine functions

$$
\begin{aligned}
& \widehat{u}_{N}(t):=\sum_{k=0}^{N-1}\left(\frac{u^{k+1}-u^{k}}{\tau}\left(t-t_{k}\right)+u^{k}\right) \chi_{\left[t_{k}, t_{k+1}\right)}(t), \quad t \in[0, T), \widehat{u}_{N}(T)=u^{N}, \\
& \widehat{M}_{N}(t)=\sum_{k=0}^{N-1}\left(\frac{M_{N}\left(t_{k+1}\right)-M_{N}\left(t_{k}\right)}{\tau}\left(t-t_{k}\right)+M_{N}\left(t_{k}\right)\right) \chi_{\left[t_{k}, t_{k+1}\right)}(t), \quad t \in[0, T] .
\end{aligned}
$$

LEMMA 2.3. There exist a generic constants $K_{i} \geqslant 0, i=1, \ldots, 6$ not depending on the discretization parameters such that

$$
\begin{gathered}
\sum_{k=0}^{N-1} \mathbb{E}\left\|u^{k+1}-u^{k}\right\|_{2}^{2} \leqslant K_{1}, \\
\max _{n=0, \ldots, N} \mathbb{E}\left\|u^{n}\right\|_{2}^{2} \leqslant K_{2}, \\
\mathbb{E} \int_{0}^{T}\left\|u_{N}^{r}\right\|_{2}^{2} \mathrm{~d} t \leqslant K_{3} \quad \text { and } \quad \mathbb{E} \int_{0}^{T}\left\|\widehat{u}_{N}\right\|_{2}^{2} \mathrm{~d} t \leqslant K_{4}, \\
\mathbb{E} \int_{0}^{T}\left\|\nabla u_{N}^{r}\right\|_{p}^{p} \mathrm{~d} t \leqslant K_{5} \quad \text { and } \quad \mathbb{E} \int_{0}^{T}\left\|\nabla \widehat{u}_{N}\right\|_{p}^{p} \mathrm{~d} t \leqslant K_{6} .
\end{gathered}
$$

Proof. We fix $n \in\{1, \ldots, N\}$, take the sum over $0, \ldots, n-1$ in $(2.10)$ to get

$$
\begin{aligned}
& \frac{1}{2} \mathbb{E}\left\|u^{n}\right\|_{2}^{2}-\frac{1}{2} \mathbb{E}\left\|u^{0}\right\|_{2}^{2}+\frac{1}{4} \sum_{k=0}^{n-1} \mathbb{E}\left\|u^{k+1}-u^{k}\right\|_{2}^{2}+C_{a}^{1} \sum_{k=0}^{n-1} \tau \mathbb{E}\left\|\nabla u^{k+1}\right\|_{p}^{p} \\
& \leqslant \sum_{k=0}^{n-1} \tau \mathbb{E}\left\|\Phi_{k}\right\|_{H S\left(L^{2}(D)\right)}^{2}+T\|\kappa\|_{1} .
\end{aligned}
$$

From (2.17) and lemma A.1 (see appendix) it follows that

$$
\begin{gathered}
\frac{1}{2} \mathbb{E}\left\|u^{n}\right\|_{2}^{2}+\frac{1}{4} \sum_{k=0}^{n-1} \mathbb{E}\left\|u^{k+1}-u^{k}\right\|_{2}^{2}+C_{a}^{1} \int_{0}^{T} \mathbb{E}\left\|\nabla u_{N}^{r}\right\|_{p}^{p} \mathrm{~d} t \\
\leqslant \frac{1}{2} \mathbb{E}\left\|u_{0}\right\|_{2}^{2}+T \mathbb{E}\|\Phi\|_{\mathcal{C}\left([0, T] ; H S\left(L^{2}(D)\right)\right.}^{2}+T\|\kappa\|_{1}
\end{gathered}
$$

and we get (2.13), (2.14) and the first inequality of (2.16). Now, from (2.14) it follows that $\mathbb{E} \int_{0}^{T}\left\|u_{N}^{r}\right\|_{2}^{2} \mathrm{~d} t \leqslant T \max _{k=1, \ldots, N} \mathbb{E}\left\|u^{k}\right\|_{2}^{2} \leqslant K_{3}$. Since there exists a constant $C \geqslant 0$ such that $\mathbb{E} \int_{0}^{T}\left\|\widehat{u}_{N}\right\|_{2}^{2} \mathrm{~d} t \leqslant C \tau \sum_{k=0}^{N} \mathbb{E}\left\|u^{k}\right\|_{2}^{2}$, we have shown both 
inequalities of (2.15). Thanks to lemma A.1 it follows that

$$
\begin{aligned}
\mathbb{E} \int_{0}^{T}\left\|\nabla \widehat{u}_{N}\right\|_{p}^{p} \mathrm{~d} t & =\mathbb{E} \sum_{k=0}^{N-1} \int_{t_{k}}^{t_{k+1}}\left\|\frac{\nabla u^{k+1}-\nabla u^{k}}{\tau}\left(t-t_{k}\right)+\nabla u^{k}\right\|_{p}^{p} \mathrm{~d} t \\
& \leqslant \mathbb{E} \sum_{k=0}^{N-1}\left(\int_{t_{k}}^{t_{k+1}} \frac{t-t_{k}}{\tau}\left\|\nabla u^{k+1}\right\|_{p}^{p} \mathrm{~d} t+\int_{t_{k}}^{t_{k+1}} \frac{t_{k+1}-t}{\tau}\left\|\nabla u^{k}\right\|_{p}^{p} \mathrm{~d} t\right) \\
& =\frac{\tau}{2} \mathbb{E} \sum_{k=0}^{N-1}\left(\left\|\nabla u^{k+1}\right\|_{p}^{p}+\left\|\nabla u^{k}\right\|_{p}^{p}\right) \leqslant C+\frac{\tau}{2}\left\|\nabla u^{0}\right\|_{p}^{p} \leqslant C
\end{aligned}
$$

and this yields the second inequality of (2.16).

Lemma 2.4. There exists a constant $K \geqslant 0$ such that

$$
\mathbb{E} \max _{n=0, \ldots, N}\left\|u^{n}\right\|_{2}^{2} \leqslant K
$$

In particular, from (2.18) it follows that there exists a possibly different, but not relabelled constant $K \geqslant 0$ such that $\mathbb{E} \sup _{t \in[0, T]}\left\|\widehat{u}_{N}\right\|_{2}^{2}<K$ and $\mathbb{E} \sup _{t \in[0, T]}\left\|u_{N}^{r}\right\|_{2}^{2}<K$ for all $N \in \mathbb{N}^{*}$.

Proof. Taking $u^{k+1}$ as a test function in (2.1), using (A2), Gauss-Green's theorem on the convection term and Hölder's and Young's inequalities we get

$$
\left\|u^{k+1}\right\|_{2}^{2}-\left\|u^{k}\right\|_{2}^{2} \leqslant 2 \tau\|\kappa\|_{1}+\left\|\Phi_{k} \Delta_{k+1} W\right\|_{2}^{2}+2\left(\Phi_{k} \Delta_{k+1} W, u^{k}\right)_{2} .
$$

Summing over $k=0, \ldots, n-1$ with $n \in\{1, \ldots, N\}$ in (2.19) and applying we get

$$
\left\|u^{n}\right\|_{2}^{2}-\left\|u^{0}\right\|_{2}^{2} \leqslant 2 T\|\kappa\|_{1}+\sum_{k=0}^{n-1}\left\|\Phi_{k} \Delta_{k+1} W\right\|_{2}^{2}+2 \sum_{k=0}^{n-1}\left(\Phi_{k} \Delta_{k+1} W, u^{k}\right)_{2}
$$

and therefore, applying lemma A.1, taking the maximum over $0, \ldots, n$ and then taking expectation we arrive at

$$
\begin{aligned}
\mathbb{E} \max _{n=0, \ldots, N}\left\|u^{n}\right\|_{2}^{2} \leqslant & \left\|u_{0}\right\|_{2}^{2}+2 T\|\kappa\|_{1}+\sum_{k=0}^{N-1} \mathbb{E}\left\|\Phi_{k} \Delta_{k+1} W\right\|_{2}^{2} \\
& +2 \mathbb{E}\left(\max _{n=1, \ldots, N} \sum_{k=0}^{n-1}\left(\Phi_{k} \Delta_{k+1} W, u^{k}\right)_{2}\right) \\
= & \left\|u_{0}\right\|_{2}^{2}+2 T\|\kappa\|_{1}+I_{1}+I_{2},
\end{aligned}
$$

where, by Itô's isometry, $I_{1}=\sum_{k=0}^{N-1} \mathbb{E}\left\|\int_{t_{k}}^{t_{k+1}} \Phi_{k} \mathrm{~d} W(t)\right\|_{2}^{2} \leqslant T \mathbb{E}\|\Phi\|_{\mathcal{C}\left([0, T] ; H S\left(L^{2}(D)\right)\right)}^{2}$. We have $\sum_{k=0}^{n-1}\left(\Phi_{k} \Delta_{k+1} W, u^{k}\right)_{2}=\int_{0}^{t_{n}}\left(\Phi_{N}, u_{N}^{l}\right)_{2} \mathrm{~d} W(t)$, where $u_{N}^{l}$ is the piecewise 
constant, left-continuous function defined by $u_{N}^{l}(t)=u^{k}$ for $t \in\left(t_{k}, t_{k+1}\right]$, $k=0, \ldots, N-1$. Thus,

$$
I_{2}=2 \mathbb{E} \max _{n=1, \ldots, N} \int_{0}^{t_{n}}\left(\Phi_{N}, u_{N}^{l}\right)_{2} \mathrm{~d} W(t) \leqslant 2 \mathbb{E} \max _{n=1, \ldots, N}\left|\int_{0}^{t_{n}}\left(\Phi_{N}, u_{N}^{l}\right)_{2} \mathrm{~d} W(t)\right| .
$$

Now, by Burkholder's and Hölder's inequalities it follows from (2.21)

$$
\begin{aligned}
I_{2} & \leqslant 6 \mathbb{E}\left(\int_{0}^{T}\left\|\left(\Phi_{N}, u_{N}^{l}\right)_{2}\right\|_{H S\left(L^{2}(D) ; \mathbb{R}\right)}^{2} \mathrm{~d} t\right)^{1 / 2} \\
& \leqslant 6 \mathbb{E}\left[\max _{n=0, \ldots, N}\left\|u^{n}\right\|_{2} \sqrt{T}\|\Phi\|_{\mathcal{C}\left([0, T] ; H S\left(L^{2}(D)\right)\right)}\right] .
\end{aligned}
$$

Using Young's inequality with $\alpha>0$ from (2.22), it follows that

$$
\begin{aligned}
I_{2} & \leqslant 3 \alpha \mathbb{E} \max _{n=0, \ldots, N}\left\|u^{n}\right\|_{2}^{2}+\frac{3 T}{\alpha} \mathbb{E}\|\Phi\|_{\mathcal{C}\left([0, T] ; H S\left(L^{2}(D)\right)\right)}^{2} \\
& \leqslant 3 \alpha \mathbb{E} \max _{n=0, \ldots, N}\left\|u^{n}\right\|_{2}^{2}+3 \alpha\left\|u_{0}\right\|_{2}^{2}+\frac{3 T}{\alpha} \mathbb{E}\|\Phi\|_{\mathcal{C}\left([0, T] ; H S\left(L^{2}(D)\right)\right)}^{2} .
\end{aligned}
$$

Plugging the estimates for $I_{1}$ and $I_{2}$ into (2.20), choosing $\alpha>0$ such that $1-3 \alpha>0$, the assertion follows.

Lemma 2.5. There exists $K \geqslant 0$ not depending on $N \in \mathbb{N}^{*}$ such that

$$
\mathbb{E} \int_{0}^{T}\left\|\frac{\mathrm{d}}{\mathrm{d} t}\left(\widehat{u}_{N}-\widehat{M}_{N}\right)\right\|_{W^{-1, p^{\prime}}(D)}^{p^{\prime}} \mathrm{d} t \leqslant K .
$$

Proof. We fix $k=0, \ldots, N-1$. For all $t \in\left(t_{k}, t_{k+1}\right)$,

$$
\frac{\mathrm{d}}{\mathrm{d} t}\left(\widehat{u}_{N}-\widehat{M}_{N}\right)=\operatorname{div}\left(a\left(x, u^{k+1}, \nabla u^{k+1}\right)+F\left(u^{k+1}\right)\right) .
$$

For any $v \in W_{0}^{1, p}(D)$, using $(A 2)$ we get

$$
\begin{aligned}
& \int_{D}\left|\left(a\left(x, u^{k+1}, \nabla u^{k+1}\right)+F\left(u^{k+1}\right)\right) \cdot \nabla v\right| \mathrm{d} x \\
& \quad \leqslant \int_{D}\left(C_{a}^{2}\left|\nabla u^{k+1}\right|^{p-1}+C_{a}^{3}\left|u^{k+1}\right|^{p-1}+g(x)+L\left|u^{k+1}\right|\right)|\nabla v| \mathrm{d} x=I_{1}+I_{2},
\end{aligned}
$$

where $L>0$ is the Lipschitz constant of $F$. Thanks to Young's and Poincaré's inequalities, a positive constant $C_{1}$ exists such that

$$
\begin{aligned}
I_{1} & =\int_{D}\left(C_{a}^{2}\left|\nabla u^{k+1}\right|^{p-1}+C_{a}^{3}\left|u^{k+1}\right|^{p-1}+g(x)\right)|\nabla v| \mathrm{d} x \\
& \leqslant \frac{C_{1}}{p^{\prime}}\left(\left\|\nabla u^{k+1}\right\|_{p}^{p}+\|g\|_{p^{\prime}}^{p^{\prime}}\right)+\frac{1}{p}\|v\|_{W_{0}^{1, p}(D)}^{p} .
\end{aligned}
$$


Since $p^{*}>p^{\prime}, L^{p^{*}}(D) \subset L^{p^{\prime}}(D)$ with continuous injection. Therefore, using Hölder's, Sobolev's and Young's inequalities, we get

$$
I_{2}=L \int_{D}\left|u^{k+1}\left\|\nabla v \mid \mathrm{d} x \leqslant \frac{C_{2}}{p}\right\| \nabla u^{k+1}\left\|_{p}^{p}+\frac{1}{p^{\prime}}\right\| v \|_{W_{0}^{1, p}(D)}^{p^{\prime}}\right.
$$

for a constant $C_{2} \geqslant 0$. Using (2.24), (2.25), (2.26) and (2.27) and taking expectation it follows that

$$
\begin{gathered}
\mathbb{E} \int_{0}^{T}\left\|\frac{\mathrm{d}}{\mathrm{d} t}\left(\widehat{u}_{N}-\widehat{M}_{N}\right)\right\|_{W^{-1, p^{\prime}(D)}}^{p^{\prime}} \mathrm{d} t \leqslant \frac{T C_{3}}{p^{\prime}}\|g\|_{p^{\prime}}+T+C_{3} \mathbb{E} \tau \sum_{k=0}^{N-1}\left\|\nabla u^{k+1}\right\|_{p}^{p} \\
=\frac{T C}{p^{\prime}}\|g\|_{p^{\prime}}+T+C \mathbb{E} \int_{0}^{T}\left\|\nabla u_{N}^{r}\right\|_{p}^{p} \mathrm{~d} t
\end{gathered}
$$

for a constant $C_{3} \geqslant 0$. Now, the assertion follows from (2.16) of lemma 2.3.

LEMMA 2.6. There exists a constant $K \geqslant 0$ such that

$$
\mathbb{E} \int_{0}^{T}\left\|u_{N}^{r}-\widehat{u}_{N}\right\|_{2}^{2} \mathrm{~d} t \leqslant K \tau
$$

Proof. We have $\mathbb{E} \int_{0}^{T}\left\|u_{N}^{r}-\widehat{u}_{N}\right\|_{2}^{2} \mathrm{~d} t=\tau / 3 \sum_{k=0}^{N-1} \mathbb{E}\left\|u^{k+1}-u^{k}\right\|_{2}^{2}$ and the assertion follows from (2.13) of lemma 2.3 .

Lemma 2.7. There exists $\gamma>0$ and $K \geqslant 0$ not depending on $N \in \mathbb{N}^{*}$ such that

$$
\mathbb{E} \sup _{k \in\{0, \ldots, N-1\}} \sup _{s \in\left[t_{k}, t_{k+1}\right]}\left\|\int_{t_{k}}^{s} \Phi_{N} \mathrm{~d} W(t)\right\|_{L^{2}(D)}^{2} \leqslant K \tau^{\gamma} .
$$

Proof. We fix $k \in\{0, \ldots, N-1\}$ and $s \in\left[t_{k}, t_{k+1}\right]$. Then we have

$$
\left\|\int_{t_{k}}^{s} \Phi_{N} \mathrm{~d} W(t)\right\|_{L^{2}(D)}^{2} \leqslant C\left\|\Phi_{k}\right\|_{L^{2}(D)}^{2}\left\|W(s)-W\left(t_{k}\right)\right\|_{U}^{2},
$$

where $C \geqslant 0$ is a constant that may change from line to line. Now, from $[\mathbf{7}],[\mathbf{1 4}$, example 2.4.1] for any $q \geqslant 1$ and $\alpha>1 / q$, it follows that

$$
\begin{aligned}
\left\|W(s)-W\left(t_{k}\right)\right\|_{U} & \leqslant C \tau^{\alpha-1 / q}\left(\int_{0}^{T} \int_{0}^{T} \frac{\|W(t)-W(r)\|_{U}^{q}}{|t-r|^{\alpha q+1}} \mathrm{~d} t \mathrm{~d} r\right)^{1 / q} \\
& =C \tau^{\alpha-1 / q} X^{1 / q}
\end{aligned}
$$

where $U$ is defined in (1.2) and $X:=\int_{0}^{T} \int_{0}^{T}\left(\left(\|W(t)-W(r)\|_{U}^{q}\right) /|t-r|^{\alpha q+1}\right) \mathrm{d} t \mathrm{~d} r$ is a real-valued random variable. Consequently,

$$
\left\|W(s)-W\left(t_{k}\right)\right\|_{U}^{2} \leqslant C \tau^{2(\alpha-1 / q)} X^{2 / q} .
$$


Plugging (2.32) into (2.31), we get

$$
\begin{aligned}
& \sup _{k \in\{0, \ldots, N-1\}} \sup _{s \in\left[t_{k}, t_{k+1}\right]}\left\|\int_{t_{k}}^{s} \Phi_{N} \mathrm{~d} W(t)\right\|_{L^{2}(D)}^{2} \\
& \leqslant C\left(\sup _{k \in\{0, \ldots, N-1\}}\left\|\Phi_{k}\right\|_{H S\left(L^{2}(D)\right)}^{2}\right) \sup _{k \in\{0, \ldots, N-1\}} \sup _{s \in\left[t_{k}, t_{k+1}\right]}\left\|W(s)-W\left(t_{k}\right)\right\|_{U}^{2} \\
& \leqslant C \tau^{\gamma} X^{2 / q} \sup _{t \in[0, T]}\|\Phi(t)\|_{L^{2}(D)}^{2}
\end{aligned}
$$

for any $q \geqslant 1, \alpha>1 / q, \gamma:=2(\alpha-1 / q)>0$. For $q>2$ with Jensen's inequality, it follows that

$$
\mathbb{E}\left(X^{2 / q}\right) \leqslant(\mathbb{E}(X))^{2 / q}=\left(\int_{0}^{T} \int_{0}^{T} \frac{\mathbb{E}\|W(t)-W(r)\|_{U}^{q}}{|t-r|^{\alpha q+1}} \mathrm{~d} t \mathrm{~d} r\right)^{2 / q} .
$$

Since $\mathbb{E}\|W(t)-W(r)\|_{U}^{q} \leqslant C|t-r|^{q / 2}$, for some $c \geqslant 0$ one gets, for $q>2$ and $\alpha \in\left(1 / q, \frac{1}{2}\right)$

$$
\mathbb{E}(X) \leqslant C \int_{0}^{T} \int_{0}^{T}|t-r|^{q / 2-\alpha q-1} \mathrm{~d} t \mathrm{~d} r<+\infty .
$$

Taking expectation in (2.33) and using (2.34) and (2.35), (2.30) holds true.

As a consequence, we can estimate the difference between the piecewise affine and the time-continuous approximations of the stochastic integral:

Lemma 2.8. There exist constants $\gamma>0, K \geqslant 0$ such that, for all $N \in \mathbb{N}^{*}$,

$$
\mathbb{E} \sup _{t \in[0, T]}\left\|M_{N}(t)-\widehat{M}_{N}(t)\right\|_{2}^{2} \leqslant K \tau^{\gamma}
$$

Proof. We fix $N \in \mathbb{N}^{*}$. For $k \in\{0, \ldots, N-1\}$ and $t \in\left[t_{k}, t_{k+1}\right)$

$$
\begin{aligned}
\left\|M_{N}(t)-\widehat{M}_{N}(t)\right\|_{2}^{2} & =\left\|\int_{t_{k}}^{t} \Phi_{N} \mathrm{~d} W(s)-\frac{t-t_{k}}{\tau} \int_{t_{k}}^{t_{k+1}} \Phi_{N} \mathrm{~d} W(s)\right\|_{2}^{2} \\
& \leqslant C\left\|\int_{t_{k}}^{t} \Phi_{N} \mathrm{~d} W(s)\right\|_{2}^{2}+\left\|\int_{t_{k}}^{t_{k+1}} \Phi_{N} \mathrm{~d} W(s)\right\|_{2}^{2}
\end{aligned}
$$

and therefore

$$
\sup _{t \in[0, T]}\left\|M_{N}(t)-\widehat{M}_{N}(t)\right\|_{2}^{2} \leqslant 2 \sup _{k=0, \ldots, N-1} \sup _{t \in\left[t_{k}, t_{k+1}\right)}\left\|\int_{t_{k}}^{t} \Phi_{N} \mathrm{~d} W(s)\right\|_{2}^{2}
$$

and the assertion follows from lemma 2.7. 


\subsection{Convergence of approximate solutions}

2.3.1. Tightness In the multiplicative noise case, the stochastic integral inherits the spatial Sobolev regularity from the solution. In the additive noise case, this is not possible and the compactness argument for processes with values in $L^{2}(D)$ is more subtle.

Lemma 2.9. $\lim _{N \rightarrow \infty} M_{N}=\lim _{N \rightarrow \infty} \widehat{M}_{N}=\int_{0}^{\cdot} \Phi \mathrm{d} W(t)$ in $L^{2}\left(\Omega ; \mathcal{C}\left([0, T] ; L^{2}(D)\right)\right.$.

Proof. From Burkholder-Davies-Gundy's inequality it follows that

$$
\mathbb{E} \sup _{[0, T]}\left\|\int_{0}^{t} \Phi_{N}-\Phi \mathrm{d} W(s)\right\|_{2}^{2} \leqslant \mathbb{E} \int_{0}^{T}\left\|\Phi_{N}-\Phi\right\|_{2}^{2} \mathrm{~d} s .
$$

Since $\Phi_{N} \rightarrow \Phi$ for $N \rightarrow \infty$ in $L^{2}\left(\Omega ; L^{2}\left(0, T ; H S\left(L^{2}(D)\right)\right)\right.$, the right-hand side of (2.37) converges to 0 when $N \rightarrow \infty$. Now, the convergence result for $\left(\widehat{M}_{N}\right)$ is a direct consequence of lemma 2.8 .

Lemma 2.10. For $N \in \mathbb{N}^{*}$, let $\mu_{N}$ be the law of

$$
X_{N}:=\left(\widehat{u}_{N}, \widehat{u}_{N}-\widehat{M}_{N}, M_{N}, \widehat{M}_{N}, \Phi_{N}, W\right) .
$$

Then, $\left(\mu_{N}\right)_{N \in \mathbb{N}^{*}}$ is a uniformly tight sequence.

Proof. By lemma 2.9, $\widehat{M}_{N}$ converges to $\int_{0}^{\cdot} \Phi \mathrm{d} W(s)$ in $L^{2}\left(\Omega ; \mathcal{C}\left([0, T] ; L^{2}(D)\right)\right)$, thus also in law. Since $\mathcal{C}\left([0, T] ; L^{2}(D)\right)$ is a Polish space, from the theorem of Prokhorov, it follows that for any $\epsilon>0$, there exists a compact set $K_{\epsilon}^{1} \subset \mathcal{C}\left([0, T], L^{2}(D)\right)$ such that $P\left[\widehat{M}_{N} \notin K_{\epsilon}^{1}\right]<\epsilon$, for all $N \in \mathbb{N}^{*}$. For the same reason, $P\left[M_{N} \notin K_{\epsilon}^{1}\right]<\epsilon$ for all $N \in \mathbb{N}^{*}$, for the same compact set. Since $\Phi_{N} \rightarrow \Phi$ in $L^{2}\left(\Omega ; L^{2}\left(0, T ; H S\left(L^{2}(D)\right)\right)\right)$ for $N \rightarrow \infty$, with similar arguments, it follows that for any $\epsilon>0$, there exists a compact set $K_{\epsilon}^{2} \subset L^{2}\left(0, T ; H S\left(L^{2}(D)\right)\right)$ such that $P\left[\Phi_{N} \notin K_{\epsilon}^{2}\right]<\epsilon$ for all $N \in \mathbb{N}^{*}$. Moreover, for any $\epsilon>0$, there exists a compact set $K_{\epsilon}^{3} \subset \mathcal{C}([0, T] ; U)$, where $U$ is defined in (1.2), such that $P\left[W \notin K_{\epsilon}^{3}\right]<\epsilon$. From lemmas 2.4 and 2.5, it follows that $\widehat{u}_{N}-\widehat{M}_{N}$ is bounded in $L^{2}\left(\Omega ; \mathcal{C}\left([0, T] ; L^{2}(D)\right)\right)$ and $(\mathrm{d} / \mathrm{d} t)\left(\widehat{u}_{N}-\widehat{M}_{N}\right)$ is bounded in $L^{p^{\prime}}\left(\Omega \times(0, T) ; W^{-1, p^{\prime}}(D)\right)$, thus $\left(\widehat{u}_{N}-\widehat{M}_{N}\right)$ is bounded in $L^{\min \left\{2, p^{\prime}\right\}}(\Omega ; \mathcal{W})$, where

$$
\mathcal{W}:=\left\{u \in L^{2}\left(0, T ; L^{2}(D)\right) \mid \frac{\mathrm{d}}{\mathrm{d} t} u \in L^{p^{\prime}}\left(0, T ; W^{-1, p^{\prime}}(D)\right)\right\}
$$

and this space is compactly embedded into $\mathcal{C}\left([0, T] ; W^{-1, p^{\prime}}(D)\right)$ by $[\mathbf{1 3}$, corollary 1$]$. Therefore, from Markov inequality, it follows that for any $\epsilon>0$, there exists $M_{\epsilon}>0$ and $K_{\epsilon}^{4}=B_{\mathcal{W}}\left(0, M_{\epsilon}\right)$ such that $P\left[\widehat{u}_{N}-\widehat{M}_{N} \notin K_{\epsilon}^{4}\right]<\epsilon$, for all $N$, with the additional information that $K_{\epsilon}^{4}$ is a relatively compact set in $\mathcal{C}\left([0, T] ; W^{-1, p^{\prime}}(D)\right)$. Thanks to lemma 2.3, $\left(\widehat{u}_{N}\right)$ is bounded in $L^{p}\left(\Omega, L^{p}\left(0, T ; W_{0}^{1, p}(D)\right)\right)$, thus again, using Markov inequality, we obtain: for any $\epsilon>0$, there exists $M>0$ such that $P\left[\left\|\widehat{u}_{N}\right\|_{L^{p}\left(0, T ; W_{0}^{1, p}(D)\right)}>M\right]<\epsilon$ for all $N \in \mathbb{N}^{*}$. Then, for $\epsilon>0$, we can define 


$$
\begin{aligned}
K_{\epsilon} & :=\bar{B}(0, M)_{L^{p}\left(0, T ; W_{0}^{1, p}(D)\right)} \times K_{\epsilon}^{4} \times K_{\epsilon}^{1} \times K_{\epsilon}^{1} \times K_{\epsilon}^{2} \times K_{\epsilon}^{3} \text { and } \\
& \widetilde{K}_{\epsilon}:=\left(\bar{B}(0, M)_{L^{p}\left(0, T ; W_{0}^{1, p}(D)\right)} \cap\left[K_{\epsilon}^{4}+K_{\epsilon}^{1}\right]\right) \times K_{\epsilon}^{4} \times K_{\epsilon}^{1} \times K_{\epsilon}^{1} \times K_{\epsilon}^{2} \times K_{\epsilon}^{3} .
\end{aligned}
$$

Consequently, if $\mu_{N}$ is the law of $X_{N}=\left(\widehat{u}_{N}, \widehat{u}_{N}-\widehat{M}_{N}, M_{N}, \widehat{M}_{N}, \Phi_{N}, W\right)$, on one hand we have

$$
\begin{array}{rl}
\mu_{N}\left(K_{\epsilon}\right)= & P\left[X_{N}^{-1}\left(K_{\epsilon}\right)\right] \\
=P & P\left\{\widehat{u}_{N} \in \bar{B}(0, M)_{L^{p}\left(0, T ; W_{0}^{1, p}(D)\right)}\right\} \cap\left\{\widehat{u}_{N}-\widehat{M}_{N} \in K_{\epsilon}^{4}\right\} \cap\left\{M_{N} \in K_{\epsilon}^{1}\right\} \\
& \left.\cap\left\{\widehat{M}_{N} \in K_{\epsilon}^{1}\right\} \cap\left\{\Phi_{N} \in K_{\epsilon}^{2}\right\} \cap\left\{W \in K_{\epsilon}^{3}\right\}\right] \\
=1 & -P\left[\left\{\widehat{u}_{N} \notin \bar{B}(0, M)_{L^{p}\left(0, T ; W_{0}^{1, p}(D)\right)}\right\} \cup\left\{\widehat{u}_{N}-\widehat{M}_{N} \notin K_{\epsilon}^{4}\right\}\right. \\
& \left.\cup\left\{M_{N} \notin K_{\epsilon}^{1}\right\} \cup\left\{\widehat{M}_{N} \notin K_{\epsilon}^{1}\right\} \cup\left\{\Phi_{N} \notin K_{\epsilon}^{2}\right\} \cup\left\{W \notin K_{\epsilon}^{3}\right\}\right] \\
\geqslant & 1-6 \epsilon
\end{array}
$$

for all $N \in \mathbb{N}^{*}$. On the other hand, since $\widehat{u}_{N}=\widehat{u}_{N}-\widehat{M}_{N}+\widehat{M}_{N}$,

$$
\begin{aligned}
\mu_{N}\left(K_{\epsilon}\right)=P & {\left[X_{N}^{-1}\left(K_{\epsilon}\right)\right] } \\
=P & {\left[\left\{\widehat{u}_{N}-\widehat{M}_{N}+\widehat{M}_{N} \in \bar{B}(0, M)_{L^{p}\left(0, T ; W_{0}^{1, p}(D)\right)}\right\} \cap\left\{\widehat{u}_{N}-\widehat{M}_{N} \in K_{\epsilon}^{4}\right\}\right.} \\
& \left.\cap\left\{M_{N} \in K_{\epsilon}^{1}\right\} \cap\left\{\widehat{M}_{N} \in K_{\epsilon}^{1}\right\} \cap\left\{\Phi_{N} \in K_{\epsilon}^{2}\right\} \cap\left\{W \in K_{\epsilon}^{3}\right\}\right] \\
=P & {\left[\left\{\widehat{u}_{N} \in \bar{B}(0, M)_{L^{p}\left(0, T ; W_{0}^{1, p}(D)\right)} \cap\left(K_{\epsilon}^{4}+K_{\epsilon}^{1}\right)\right\} \cap\left\{\widehat{u}_{N}-\widehat{M}_{N} \in K_{\epsilon}^{4}\right\}\right.} \\
& \left.\cap\left\{M_{N} \in K_{\epsilon}^{1}\right\} \cap\left\{\widehat{M}_{N} \in K_{\epsilon}^{1}\right\} \cap\left\{\Phi_{N} \in K_{\epsilon}^{2}\right\} \cap\left\{W \in K_{\epsilon}^{3}\right\}\right] \\
= & P\left(X_{N}^{-1}\left(\widetilde{K}_{\epsilon}\right)\right)=\mu_{N}\left(\widetilde{K}_{\epsilon}\right) .
\end{aligned}
$$

Note that by [13, lemma 9], $\bar{B}(0, M)_{L^{p}\left(0, T ; W_{0}^{1, p}(D)\right)} \cap\left(K_{\epsilon}^{4}+K_{\epsilon}^{1}\right)$ is relatively compact in $L^{2}\left(0, T, L^{2}(D)\right)$ since $K_{\epsilon}^{4}+K_{\epsilon}^{1}$ is relatively compact in $L^{2}\left(0, T ; W^{-1, p^{\prime}}(D)\right)$ and $W_{0}^{1, p}(D)$ is compactly embedded into $L^{2}(D)$. Thus, $\left(\mu_{N}\right)$ is a uniformly tight sequence.

Proposition 2.11. The sequence $\left(\nu_{N}\right)$ of laws induced by

$$
Y_{N}=\left(u_{N}^{r}-\widehat{u}_{N}, \widehat{u}_{N}, \widehat{u}_{N}-\widehat{M}_{N}, M_{N}, \widehat{M}_{N}, \Phi_{N}, W\right)
$$

on the product space

$$
\begin{aligned}
\mathcal{X}:= & L^{2}\left(Q_{T}\right) \times L^{2}\left(Q_{T}\right) \times \mathcal{C}\left([0, T] ; W^{-1, p^{\prime}}(D)\right) \times \mathcal{C}\left([0, T] ; L^{2}(D)\right) \\
& \times \mathcal{C}\left([0, T] ; L^{2}(D)\right) \times L^{2}\left(0, T ; H S\left(L^{2}(D)\right)\right) \times \mathcal{C}([0, T] ; U),
\end{aligned}
$$

where $U$ is defined in (1.2), is tight and therefore, passing to a not relabelled subsequence if necessary, there exist probability measures $\nu_{1}$ on $L^{2}\left(Q_{T}\right), \nu_{2}$ on 
$\mathcal{C}\left([0, T] ; W^{-1, p^{\prime}}(D)\right)$ and a probability measure

$$
\nu:=\left(0, \nu_{1}, \nu_{2}, \mathcal{L}\left(\int_{0}^{.} \Phi \mathrm{d} W(s)\right), \mathcal{L}\left(\int_{0}^{\cdot} \Phi \mathrm{d} W(s)\right), \mathcal{L}(\Phi), \mathcal{L}(W)\right)
$$

on $\mathcal{X}$ such that $\nu_{N} \Rightarrow \nu$, i.e., $\lim _{N \rightarrow \infty} \int_{\mathcal{X}} f \mathrm{~d} \nu_{N}=\int_{\mathcal{X}} f \mathrm{~d} \nu$ for all $f \in \mathcal{C}_{b}(\mathcal{X})$.

Proof. Note that for all $N \in \mathbb{N}^{*}, \nu_{N}=\left(\mathcal{L}\left(u_{N}^{r}-\widehat{u}_{N}\right), \mu_{N}\right)$ and the tightness of $\left(\mu_{N}\right)$ follows from lemma 2.10. The tightness of $\left(\mathcal{L}\left(u_{N}^{r}-\widehat{u}_{N}\right)\right)$ is a direct consequence of lemma 2.6 and the converse part of Prokhorov's theorem.

2.3.2. Compactness Now, we are in position to apply the theorem of Skorokhod: by Appendix A.3, there exists a new probability space $\left(\Omega^{\prime}, \mathcal{F}^{\prime}, P^{\prime}\right)^{1}$ such that, passing to a subsequence if necessary, $\left(Y_{N}\right)$ converges almost surely in $\mathcal{X}$. Without changing the notation of random variables with the same law in order not to overload the presentation, the semi-implicit Euler scheme (2.1) is satisfied on $\Omega^{\prime}{ }^{2}$ Moreover, remark A.1 and in particular the a priori estimates developed in lemmas 2.2 to 2.6 hold true on $\left(\Omega^{\prime}, \mathcal{F}^{\prime}, P^{\prime}\right)$. Thus, on $\left(\Omega^{\prime}, \mathcal{F}^{\prime}, P^{\prime}\right)$,

- there exists a $L^{2}\left(Q_{T}\right)$-valued random variable $u_{\infty}$ such that $\mathcal{L}\left(u_{\infty}\right)=\mathcal{L}\left(\nu_{1}\right)$ and $\lim _{N \rightarrow \infty} \widehat{u}_{N}=u_{\infty}$ in $L^{2}\left(Q_{T}\right)$ a.s. in $\Omega^{\prime}$. Then, lemma 2.4 and Vitali's theorem yield the convergence of $\left(\widehat{u}_{N}\right)$ to $u_{\infty}$ in $L^{\ell}\left(\Omega^{\prime} ; L^{2}\left(Q_{T}\right)\right)$ for all $1 \leqslant \ell<2$.

- $\left(u_{N}^{r}-\widehat{u}_{N}\right)$ converges a.s. to 0 in $L^{2}\left(Q_{T}\right)$ and one proves similarly, or by using (2.29), that $\lim _{N \rightarrow \infty} u_{N}^{r}=u_{\infty}$ in $L^{2}\left(Q_{T}\right)$ a.s. in $\Omega^{\prime}$ and in $L^{\ell}\left(\Omega^{\prime} ; L^{2}\left(Q_{T}\right)\right)$ for all $1 \leqslant \ell<2$.

- In particular, the above convergence holds in $L^{\ell}\left(\Omega^{\prime} \times(0, T) ; L^{2}(D)\right)$ and, up to a subsequence if necessary, $\left(u_{N}^{r}(\omega, t)\right)$ converges to $u_{\infty}(\omega, t)$ in $L^{2}(D)$, a.e. in $\Omega^{\prime} \times(0, T)$.

- There exists a $\mathcal{C}\left([0, T] ; L^{2}(D)\right)$-valued random variable $M_{\infty}$ such that $\mathcal{L}\left(M_{\infty}\right)$ $=\mathcal{L}\left(\int_{0}^{\cdot} \Phi d W(s)\right)$ and $\lim _{N \rightarrow \infty} \widehat{M}_{N}=\lim _{N \rightarrow \infty} M_{N}=M_{\infty}$ in $\mathcal{C}\left([0, T] ; L^{2}(D)\right)$ a.s. in $\Omega^{\prime}$. Using remark A.1, lemma 2.9 and the lemma of Brezis-Lieb (see appendix), we also obtain the convergence in $L^{2}\left(\Omega^{\prime} ; \mathcal{C}\left([0, T] ; L^{2}(D)\right)\right)$.

- There exists a $\mathcal{C}\left([0, T] ; H S\left(L^{2}(D)\right)\right)$-valued random variable $\Phi_{\infty}$ such that $\mathcal{L}\left(\Phi_{\infty}\right)=\mathcal{L}(\Phi)$ and $\lim _{N \rightarrow \infty} \Phi_{N}=\Phi_{\infty}$ in $L^{2}\left(0, T ; H S\left(L^{2}(D)\right)\right)$ a.s. in $\Omega^{\prime}$. Again, remark A.1 and Brezis-Lieb's lemma yield the convergence in $L^{2}\left(\Omega^{\prime} ; L^{2}\left(0, T ; H S\left(L^{2}(D)\right)\right)\right)$.

- Further, there exists a $\mathcal{C}\left([0, T] ; W^{-1, p^{\prime}}(D)\right)$-valued random variable $B_{\infty}$ on $\Omega^{\prime}$ such that $\mathcal{L}\left(B_{\infty}\right)=\nu_{2}$ and $\lim _{N \rightarrow \infty}\left(\widehat{u}_{N}-\widehat{M}_{N}\right)=B_{\infty}$ in $\mathcal{C}\left([0, T] ; W^{-1, p^{\prime}}(D)\right)$ a.s. in $\Omega^{\prime}$ and in $L^{\tilde{\ell}}\left(\Omega, \mathcal{C}\left([0, T] ; W^{-1, p^{\prime}}(D)\right)\right)$ for any $1 \leqslant \tilde{\ell}<\min \left(2, p^{\prime}\right)$ by Vitali's theorem. Thanks to the previous convergence results, $\left(\widehat{u}_{N}\right)=\left(\widehat{u}_{N}-\right.$ $\left.\widehat{M}_{N}+\widehat{M}_{N}\right)$ converges a.s. in $\mathcal{C}\left([0, T] ; W^{-1, p^{\prime}}(D)\right)$. Thus, $B_{\infty}=u_{\infty}-M_{\infty}$,

\footnotetext{
${ }^{1}$ This can be $(0,1)$ with the Lebesgue $\sigma$-field and measure and expectation denoted $\mathbb{E}^{\prime}$.

${ }^{2}$ Note that, because of Skorokhod's theorem, $W_{N}$ needs to be indexed by $N$ in $\Omega^{\prime}$.
} 
$u_{\infty} \in \mathcal{C}\left([0, T] ; W^{-1, p^{\prime}}(D)\right)$ and lemma 2.4 with Vitali's theorem yield the convergence of $\widehat{u}_{N}$ to $u_{\infty}$ in $L^{\ell}\left(\Omega^{\prime} ; \mathcal{C}\left([0, T] ; W^{-1, p^{\prime}}(D)\right)\right)$ for all $1 \leqslant \ell<2$.

- Finally, there exists a random variable $W_{\infty}$ with values in $\mathcal{C}([0, T] ; U)$, such that $\mathcal{L}\left(W_{\infty}\right)=\mathcal{L}(W), \lim _{N \rightarrow \infty} W_{N}=W_{\infty}$ in $\mathcal{C}([0, T] ; U)$ a.s. in $\Omega^{\prime}$ and in $L^{2}\left(\Omega^{\prime} ; \mathcal{C}([0, T] ; U)\right)$ thanks to remark A.1 and Brezis-Lieb's lemma.

2.3.3. First identifications at the limits Note that $W_{N}$ is $\left(\mathcal{F}_{t}^{N}\right)$-measurable, where $\left(\mathcal{F}_{t}^{N}\right)$ is the filtration generated by $W_{N}$ and, for any $h \in U, t \in[0, T], 0 \leqslant s \leqslant t$ and all $\psi \in \mathcal{C}_{b}(\mathcal{C}([0, s] ; U))$

$$
\mathbb{E}^{\prime}\left[\left(W_{N}(t)-W_{N}(s), h\right)_{U} \psi\left(W_{N}\right)\right]=\mathbb{E}\left[(W(t)-W(s), h)_{U} \psi(W)\right]=0,
$$

since $W$ is a $\left(\mathcal{F}_{t}\right)$-martingale. Thus, $W_{N}$ is a $\left(\mathcal{F}_{t}^{N}\right)$-martingale satisfying $W_{N}(0)=0$ for all $N \in \mathbb{N}^{*}$. With similar arguments, one can show that $\ll W_{N} \gg_{t}=t Q$ for all $t \in[0, T]$ and all $N \in \mathbb{N}^{*}$, where $Q$ is the covariance operator of $W$. From Levy's theorem (see [3, proposition 3.11, p. 75]) it follows that $W_{N}$ is a cylindrical Wiener process with values in $L^{2}(D)$ with respect to $\left(\mathcal{F}_{t}^{N}\right)$. In particular $\left(\Omega^{\prime}, \mathcal{F}^{\prime},\left(\mathcal{F}_{t}^{N}\right), \mathbb{P}^{\prime}, W_{N}\right)$ is a stochastic basis in the sense of $[\mathbf{4}$, lemma 2.1, p. 1126]. We recall that we recovered the time discretization scheme in $\Omega^{\prime}$ and therefore the following approximate equations hold true: for all $N \in \mathbb{N}^{*}$, we have

$$
\left(\widehat{M}_{N}-\widehat{u}_{N}\right)(t)-u_{0}-\int_{0}^{t} \operatorname{div}\left(a\left(x, u_{N}^{r}, \nabla u_{N}^{r}\right)+F\left(u_{N}^{r}\right)\right) \mathrm{d} s=0
$$

in $W^{-1, p^{\prime}}(D)$ for all $t \in[0, T]$, a.s. in $\Omega^{\prime}$. Thanks to lemma 2.3, we have the following weak convergences:

LEMMA 2.12. Up to a subsequence, $u_{N}^{r} \rightarrow u_{\infty}$ weakly in $L^{p}\left(\Omega^{\prime} ; L^{p}\left(0, T ; W_{0}^{1, p}(D)\right)\right)$ for $N \rightarrow \infty$. Moreover, there exists $G \in L^{p^{\prime}}\left(\Omega^{\prime} \times Q_{T}\right)$ such that $a\left(x, u_{N}^{r}, \nabla u_{N}^{r}\right) \rightarrow G$ weakly in $L^{p^{\prime}}\left(\Omega^{\prime} \times Q_{T}\right)$.

Now we are ready to pass to the limit in the approximate equations:

Proposition 2.13.

$$
u_{\infty}(t)=u_{0}+\int_{0}^{t} \operatorname{div}\left(G+F\left(u_{\infty}\right)\right) \mathrm{d} s+M_{\infty}(t)
$$

holds in $L^{2}(D)$ a.s. in $\Omega^{\prime}$ for all $t \in[0, T]$.

REMARK 2.1. Using lemma 2.4 , one gets that $\left(\widehat{u}_{N}\right)$ is bounded in $L^{2}\left(\Omega^{\prime} ; L^{\infty}(0, T\right.$; $\left.\left.L^{2}(D)\right)\right)$, thus in $L_{w}^{2}\left(\Omega^{\prime} ; L^{\infty}\left(0, T ; L^{2}(D)\right)\right)$ where $w$ stands for the weak-* measurability. Since [5, theorem 8.20.3, p. 606]

$$
L_{w}^{2}\left(\Omega^{\prime} ; L^{\infty}\left(0, T ; L^{2}(D)\right)\right) \simeq\left(L^{2}\left(\Omega^{\prime} ; L^{1}\left(0, T ; L^{2}(D)\right)\right)\right)^{*},
$$

the theorem of Banach-Alaoglu yields $u_{\infty} \in L_{w}^{2}\left(\Omega^{\prime} ; L^{\infty}\left(0, T ; L^{2}(D)\right)\right)$. On the other hand, since $\widehat{u}_{N}$ converges to $u_{\infty}$ in $L^{\ell}\left(\Omega^{\prime} ; \mathcal{C}\left([0, T] ; W^{-1, p^{\prime}}(D)\right)\right)$, it follows that $u_{\infty}$ is a random variable with values in the space of weakly continuous functions 
$C_{w}\left([0, T] ; L^{2}(D)\right)\left[\mathbf{1 5}\right.$, lemma 1.4, p. 263]. In particular, $u_{\infty}(t) \in L^{2}(D)$ a.s. in $\Omega^{\prime}$ for all $t \in[0, T]$. Therefore, (2.39) holds in $L^{2}(D)$, a.s. in $\Omega^{\prime}$, for all $t \in[0, T]$.

Proof of proposition 2.13. We fix $\psi \in W_{0}^{1, p}(D), \xi \in \mathcal{D}(0, T), A \in \mathcal{F}^{\prime}$ and use $\psi \xi \chi_{A}$ as a test function in (2.38). Integrating over $\Omega^{\prime}$ we arrive at

$$
\begin{aligned}
& \int_{A} \int_{0}^{T}\left\langle\frac{\mathrm{d}}{\mathrm{d} t}\left(\widehat{u}_{N}-\widehat{M}_{N}\right)(t), \psi\right\rangle_{W^{-1, p^{\prime}}(D), W_{0}^{1, p}(D)} \xi \mathrm{d} t \mathrm{~d} P^{\prime} \\
& +\int_{A} \int_{0}^{T} \int_{D}\left(a\left(x, u_{n}^{r}, \nabla u_{N}^{r}\right)+F\left(u_{N}^{r}\right)\right) \cdot \nabla \psi \xi \mathrm{d} x \mathrm{~d} t \mathrm{~d} P^{\prime}=0 .
\end{aligned}
$$

Let us write $(2.40)$ as $I_{1}+I_{2}+I_{3}=0$, where

$$
\begin{aligned}
I_{1} & =\int_{A} \int_{0}^{T}\left\langle\frac{\mathrm{d}}{\mathrm{d} t}\left(\widehat{u}_{N}-\widehat{M}_{N}\right)(t), \psi\right\rangle_{W^{-1, p^{\prime}}(D), W_{0}^{1, p}(D)} \xi \mathrm{d} t \mathrm{~d} P^{\prime} \\
& =\int_{A} \int_{0}^{T} \frac{\mathrm{d}}{\mathrm{d} t}\left\langle\left(\widehat{u}_{N}-\widehat{M}_{N}\right)(t), \psi\right\rangle_{W^{-1, p^{\prime}}(D), W_{0}^{1, p}(D)} \xi \mathrm{d} t \mathrm{~d} P^{\prime} \\
& =-\int_{A} \int_{0}^{T}\left\langle\left(\widehat{u}_{N}-\widehat{M}_{N}\right)(t), \psi\right\rangle_{W^{-1, p^{\prime}}(D), W_{0}^{1, p}(D)} \xi_{t} \mathrm{~d} t \mathrm{~d} P^{\prime}, \\
I_{2} & =\int_{A} \int_{0}^{T} \int_{D} a\left(x, u_{n}^{r}, \nabla u_{N}^{r}\right) \cdot \nabla \psi \xi \mathrm{d} x \mathrm{~d} t \mathrm{~d} P^{\prime}, \\
I_{3} & =\int_{A} \int_{0}^{T} \int_{D} F\left(u_{N}^{r}\right) \cdot \nabla \psi \xi \mathrm{d} x \mathrm{~d} t \mathrm{~d} P^{\prime} .
\end{aligned}
$$

Since $\widehat{u}_{N}-\widehat{M}_{N}$ converges to $u_{\infty}-M_{\infty}$ in $L^{\tilde{\ell}}\left(\Omega^{\prime} ; \mathcal{C}\left([0, T] ; W^{-1, p^{\prime}}(D)\right)\right)$, it follows that

$$
\lim _{N \rightarrow \infty} I_{1}=\int_{A} \int_{0}^{T}\left\langle\left(\widehat{u}_{\infty}-\widehat{M}_{\infty}\right)(t), \psi\right\rangle_{W^{-1, p^{\prime}}(D), W_{0}^{1, p}(D)} \xi_{t} \mathrm{~d} x \mathrm{~d} t \mathrm{~d} P^{\prime} .
$$

We recall that, by remark 2.1, $\left(u_{\infty}-M_{\infty}\right)(t) \in L^{2}(D)$ for all $t \in[0, T]$, a.s. in $\Omega^{\prime}$. Thus

$$
\lim _{N \rightarrow \infty} I_{1}=\int_{A} \int_{0}^{T} \int_{D}\left(u_{\infty}-M_{\infty}\right) \partial_{t} \xi \psi \mathrm{d} x \mathrm{~d} t \mathrm{~d} P^{\prime} .
$$

According to lemma $2.12 a\left(x, u_{N}^{r}, \nabla u_{N}^{r}\right) \rightarrow G$ weakly in $L^{p^{\prime}}\left(\hat{\Omega} \times Q_{T}\right)^{d}$ and therefore

$$
\lim _{N \rightarrow \infty} I_{2}=\int_{A} \int_{0}^{T} \int_{D} G \cdot \nabla \psi \xi \mathrm{d} x \mathrm{~d} t \mathrm{~d} P^{\prime}
$$

$L \geqslant 0$ being the Lipschitz constant of $F$, we have, thanks to (2.16)

$$
\mathbb{E}^{\prime} \int_{0}^{T}\left\|F\left(u_{N}^{r}\right)\right\|_{W_{0}^{1, p}(D)}^{p} \mathrm{~d} t \leqslant L \mathbb{E}^{\prime} \int_{0}^{T}\left\|\nabla u_{N}^{r}\right\|_{p}^{p} \mathrm{~d} t \leqslant C
$$

where $C \geqslant 0$ is a constant not depending on $N \in \mathbb{N}^{*}$. 
Consequently, there exists $H \in L^{p}\left(\Omega^{\prime} ; L^{p}\left(0, T ;\left(W_{0}^{1, p}(D)\right)^{d}\right)\right)$ such that $F\left(u_{N}^{r}\right) \rightarrow$ $H$ weakly in $L^{p}\left(\Omega^{\prime} ; L^{p}\left(0, T ; W_{0}^{1, p}(D)^{d}\right)\right)$. Since $u_{N}^{r} \rightarrow u_{\infty}$ in $L^{1}\left(\Omega^{\prime} \times Q_{T} ; L^{2}(D)^{d}\right)$, $F\left(u_{n}^{r}\right) \rightarrow F\left(u_{\infty}\right)$ holds in $L^{1}\left(\Omega^{\prime} \times Q_{T} ; L^{2}(D)\right)$ and $H=F\left(u_{\infty}\right)$. Now, by Sobolev embedding, it follows that $F\left(u_{N}^{r}\right) \rightarrow F\left(u_{\infty}\right)$ weakly in $L^{p}\left(\Omega^{\prime} ; L^{p}\left(0, T ; L^{p^{*}}(D)^{d}\right)\right)$. Since $p^{\prime}<p^{*}$, we conclude that $F\left(u_{N}^{r}\right) \rightarrow F\left(u_{\infty}\right)$ weakly in $L^{p}\left(\Omega^{\prime} ; L^{p}(0, T\right.$; $\left.\left.L^{p^{\prime}}(D)^{d}\right)\right)$. Therefore, it follows that

$$
\lim _{N \rightarrow \infty} I_{3}=\int_{A} \int_{0}^{T} \int_{D} F\left(u_{\infty}\right) \cdot \nabla \psi \xi \mathrm{d} x \mathrm{~d} t \mathrm{~d} P^{\prime} .
$$

Now, from (2.41)-(2.43) it follows that

$$
-\int_{A} \int_{0}^{T} \int_{D}\left(u_{\infty}-M_{\infty}\right) \xi_{t} \psi+\left(G+F\left(u_{\infty}\right)\right) \cdot \nabla \psi \xi \mathrm{d} x \mathrm{~d} t \mathrm{~d} P^{\prime}=0
$$

for all $A \in \hat{\mathcal{F}}, \xi \in \mathcal{D}(0, T)$ and all $\psi \in W_{0}^{1, p}(D)$.

From (2.44) it follows that $(\mathrm{d} / \mathrm{d} t)\left(u_{\infty}-M_{\infty}\right)-\operatorname{div}\left(G+F\left(u_{\infty}\right)\right)=0$ in $\left.L^{p^{\prime}}\left(0, T ; W^{-1, p^{\prime}}(D)\right)\right)$, a.s. in $\Omega^{\prime}$. We recall that $u_{\infty} \in \mathcal{C}\left([0, T] ; W^{-1, p^{\prime}}(D)\right)$ a.s. in $\Omega^{\prime}$ and since, a.s. in $\Omega^{\prime}, M_{\infty} \in \mathcal{C}\left([0, T] ; L^{2}(D)\right)$, we have

$$
u_{\infty}(t)-u_{\infty}(0)-\int_{0}^{t} \operatorname{div}\left(G+F\left(u_{\infty}\right)\right) \mathrm{d} s=M_{\infty}(t)
$$

for all $t \in[0, T]$ in $L^{2}(D)$ a.s. in $\Omega^{\prime}$.

REMARK 2.2. Note that for any $t \in[0, T]$,

$$
\delta_{t}: L^{2}\left(\Omega^{\prime} ; \mathcal{C}\left([0, T] ; W^{-1, p^{\prime}}(D)\right)\right) \rightarrow L^{2}\left(\Omega^{\prime} ; W^{-1, p^{\prime}}(D)\right), \quad u \mapsto u(t)
$$

is continuous and therefore $u_{\infty}(0)=\lim _{N \rightarrow \infty} \widehat{u}_{N}(0)=u_{0}$ in $L^{2}\left(\Omega^{\prime} ; W^{-1, p^{\prime}}(D)\right)$.

\subsection{Martingale identification argument}

We denote the augmentation of the filtration $\sigma\left(W_{\infty}(s), \Phi_{\infty}(s), u_{\infty}(s)\right)_{0 \leqslant s \leqslant t}, t \in$ $[0, T]$ by $\left(\mathcal{F}_{t}^{\infty}\right)$. In the following two lemmas, we will show that $W_{\infty}$ is a cylindrical Wiener process with values in $L^{2}(D)$ with respect to $\left(\mathcal{F}_{t}^{\infty}\right)$. To this end, we first show that $W_{\infty}$ is a $\left(\mathcal{F}_{t}^{\infty}\right)$-martingale. Since $u_{\infty}$ is in $\mathcal{C}\left([0, T] ; W^{-1, p^{\prime}}(D)\right)$ a.s. in $\Omega^{\prime}$, it is a stochastic process and therefore $\left(\mathcal{F}_{t}^{\infty}\right)$ is well defined.

Lemma 2.14. $W_{\infty}$ is a $\left(\mathcal{F}_{t}^{\infty}\right)$-martingale.

Proof. By definition of $\left(\mathcal{F}_{t}^{\infty}\right), W_{\infty}$ is adapted to $\left(\mathcal{F}_{t}^{\infty}\right)$. For all $t \in[0, T], 0 \leqslant s \leqslant t$, $\psi \in \mathcal{C}_{b}\left(\mathcal{C}([0, s] ; U) \times L^{2}\left(0, s ; H S\left(L^{2}(D)\right)\right) \times L^{2}((0, s) \times D)\right.$ and $h \in U$

$$
\begin{aligned}
\mathbb{E}^{\prime} & {\left[\left(W_{\infty}(t)-W_{\infty}(s), h\right)_{U} \psi\left(W_{\infty}, \Phi_{\infty}, u_{\infty}\right)\right] } \\
& =\lim _{N \rightarrow \infty} \mathbb{E}^{\prime}\left[\left(W_{N}(t)-W_{N}(s), h\right)_{U} \psi\left(W_{N}, \Phi_{N}, u_{N}^{r}\right)\right] \\
& =\lim _{N \rightarrow \infty} \mathbb{E}\left[(W(t)-W(s), h)_{U} \psi\left(W, \Phi_{N}, u_{N}^{r}\right)\right]=0
\end{aligned}
$$

since $\Phi_{N}$ and $u_{N}^{r}$ are $\left(\mathcal{F}_{t}\right)$-adapted processes for all $N \in \mathbb{N}^{*}$ and $W$ is a $\left(\mathcal{F}_{t}\right)$-martingale. 
Lemma 2.15. $W_{\infty}$ is a cylindrical Wiener process with values in $L^{2}(D)$ with respect to $\left(\mathcal{F}_{t}^{\infty}\right)$.

Proof. Since we already know that $W_{\infty}$ is a $\left(\mathcal{F}_{t}^{\infty}\right)$-martingale with $W_{\infty}(0)=0$, according to $[\mathbf{3}$, theorem 4.4 , p. 89$]$, it is left to show that

$$
\ll W_{\infty} \gg_{t}=t Q \quad \text { for all } t \in[0, T]
$$

where $Q$ is the covariance operator of $W$. Recall that $\ll W_{N} \gg_{t}=t Q$ for all $t \in[0, T]$ and all $N \in \mathbb{N}^{*}$. Let $U$ be the Hilbert space defined in (1.2) and $\left(g_{l}\right)$ be an orthonormal basis of $U$. For all $t \in[0, T], 0 \leqslant s \leqslant t, \psi \in \mathcal{C}_{b}(\mathcal{C}([0, s] ; U) \times$ $L^{2}\left(0, s ; H S\left(L^{2}(D)\right)\right) \times L^{2}((0, s) \times D)$ and $n, m \in \mathbb{N}^{*}$, combining the convergence results from the previous section with the dominated convergence theorem of Lebesgue, it follows that

$$
\begin{aligned}
\mathbb{E}^{\prime}[ & \left.\left(\left(W_{\infty}, g_{n}, g_{m}\right)(t)-\left(W_{\infty}, g_{n}, g_{m}\right)(s)-\left((t-s) Q\left(g_{n}\right), g_{m}\right)_{U}\right) \psi\left(W_{\infty}, \Phi_{\infty}, u_{\infty}\right)\right] \\
= & \lim _{N \rightarrow \infty} \mathbb{E}^{\prime}\left[\left(\left(W_{N}, g_{n}, g_{m}\right)(t)-\left(W_{N}, g_{n}, g_{m}\right)(s)\right.\right. \\
& \left.\left.-\left((t-s) Q\left(g_{n}\right), g_{m}\right)_{U}\right) \psi\left(W_{N}, \Phi_{N}, u_{N}^{r}\right)\right] \\
= & \lim _{N \rightarrow \infty} \mathbb{E}\left[\left(\left(W, g_{n}, g_{m}\right)(t)-\left(W, g_{n}, g_{m}\right)(s)\right.\right. \\
& \left.\left.\quad-\left((t-s) Q\left(g_{n}\right), g_{m}\right)_{U}\right) \psi\left(W, \Phi_{N}, u_{N}^{r}\right)\right]=0,
\end{aligned}
$$

where $\left(W, g_{n}, g_{m}\right)(r):=\left(W(r), g_{n}\right)_{U}\left(W(r), g_{m}\right)_{U}$ for $W(r) \in U, r \in[0, T]$, thus (2.46) holds true. In particular, $\left(\Omega^{\prime}, \mathcal{F}^{\prime},\left(\mathcal{F}_{t}^{\infty}\right), \mathbb{P}^{\prime}, W_{\infty}\right)$ is a stochastic basis in the sense of $[\mathbf{4}$, lemma 2.1, p. 1126].

From $\S$ A.3 of the appendix it follows that $\Phi_{N}$ is a $\left(\mathcal{F}_{t}^{N}\right)$-predictable process with values in $H S\left(L^{2}(D)\right)$ and that $M_{N}(t)=\int_{0}^{t} \Phi_{N} d W_{N}(s)$. In order to pass to the limit, we recall the following lemma:

Lemma 2.16. [4, lemma 2.1, p. 1126] Let $(\Omega, \mathcal{F}, P)$ be a fixed probability space, $W$ a cylindrical Wiener process on $(\Omega, \mathcal{F}, P)$ with values in $L^{2}(D)$ with respect to a given filtration $\left(\mathcal{F}_{t}\right)$ and $X$ a separable Hilbert space. Consider a sequence of stochastic bases $S_{n}=\left(\Omega, \mathcal{F},\left(\mathcal{F}_{t}^{n}\right), P, W_{n}\right)$, that is a sequence so that each $W_{n}$ is a cylindrical Wiener process with values in $L^{2}(D)$ with respect to $\left(\mathcal{F}_{t}^{n}\right)$. Assume that $\left(G_{n}\right)$ is a collection of $H S\left(L^{2}(D) ; X\right)$-valued, $\left(\mathcal{F}_{t}^{n}\right)$-predictable processes such that $G_{n} \in L^{2}\left(0, T ; H S\left(L^{2}(D) ; X\right)\right)$ a.s. in $\Omega$ and $G \in L^{2}\left(0, T ; H S\left(L^{2}(D) ; X\right)\right)$ is $\left(\mathcal{F}_{t}\right)$ predictable. If $W_{n} \rightarrow W$ in probability in $\mathcal{C}([0, T] ; U)$, where $U$ is given in $(1.2)$ and $G_{n} \rightarrow G$ in probability in $L^{2}\left(0, T ; H S\left(L^{2}(D) ; X\right)\right)$, then

$$
\lim _{n \rightarrow \infty} \int_{0}^{\cdot} G_{n} \mathrm{~d} W_{n}(s)=\int_{0}^{\cdot} G \mathrm{~d} W(s)
$$

in probability in $L^{2}(0, T ; X)$. 
Consequently, we have $M_{\infty}(t)=\int_{0}^{t} \Phi_{\infty} \mathrm{d} W_{\infty}(s)$ for all $t \in[0, T]$, a.s. in $\Omega^{\prime}$ and therefore

$$
u_{\infty}(t)=u_{0}+\int_{0}^{t} \operatorname{div}\left(G+F\left(u_{\infty}\right)\right) \mathrm{d} s+\int_{0}^{t} \Phi_{\infty} \mathrm{d} W_{\infty}(s)
$$

in $W^{-1, p^{\prime}}(D)$, for all $t \in[0, T]$, a.s. in $\Omega^{\prime}$. By lemma 2.4 and Itô's formula (see, e.g. $[\mathbf{1 0}$, theorem 4.2 .5$])$, it follows that $u_{\infty}$ is a square-integrable, $\left(\mathcal{F}_{t}^{\infty}\right)$-adapted, continuous process with values in $L^{2}(D)$, and $(2.47)$ holds in $L^{2}(D)$ a.s. in $\Omega^{\prime}$.

\subsection{Monotonicity argument}

LEMMA 2.17. $G=a\left(x, u_{\infty}, \nabla u_{\infty}\right)$ in $L^{p^{\prime}}\left(\Omega^{\prime} \times Q_{T}\right)^{d}$.

Proof. Taking $u^{k+1}$ as a test function in the discretized equation (2.1) in $\Omega^{\prime}$, using Gauss-Green's theorem for the convection term and taking expectation we get

$$
\begin{aligned}
& \frac{1}{2} \mathbb{E}^{\prime}\left(\left\|u^{k+1}\right\|_{2}^{2}-\left\|u^{k}\right\|_{2}^{2}+\left\|u^{k+1}-u^{k}\right\|_{2}^{2}\right)+\tau \mathbb{E}^{\prime} \int_{D} a\left(x, u^{k+1}, \nabla u^{k+1}\right) \cdot \nabla u^{k+1} \mathrm{~d} x \\
& \quad=\mathbb{E}^{\prime}\left(M_{N}\left(t_{k+1}\right)-M_{N}\left(t_{k}\right), u^{k+1}\right)_{2} .
\end{aligned}
$$

Since $\mathbb{E}^{\prime}\left(M_{N}\left(t_{k+1}\right)-M_{N}\left(t_{k}\right), u^{k}\right)_{2}=0$, using Young's inequality in (2.48) we get

$$
\begin{aligned}
& \mathbb{E}^{\prime}\left(\left\|u^{k+1}\right\|_{2}^{2}-\left\|u^{k}\right\|_{2}^{2}\right)+2 \tau \mathbb{E}^{\prime} \int_{D} a\left(x, u^{k+1}, \nabla u^{k+1}\right) \cdot \nabla u^{k+1} \mathrm{~d} x \\
& \quad \leqslant \mathbb{E}^{\prime}\left\|M_{N}\left(t_{k+1}\right)-M_{N}\left(t_{k}\right)\right\|_{2}^{2} .
\end{aligned}
$$

Summing over $k=0, \ldots, N-1$ in (2.49) it follows that

$$
\begin{aligned}
\mathbb{E}^{\prime} & {\left[\left\|\widehat{u}_{N}(T)\right\|_{2}^{2}-\left\|u^{0}\right\|_{2}^{2}+2 \int_{0}^{T} \int_{D} a\left(x, u_{N}^{r}, \nabla u_{N}^{r}\right) \cdot \nabla u_{N}^{r} \mathrm{~d} x \mathrm{~d} t\right] } \\
& -\sum_{k=0}^{N-1} \mathbb{E}^{\prime}\left\|\int_{t_{k}}^{t_{k+1}} \Phi_{N} \mathrm{~d} W_{N}(t)\right\|_{2}^{2} \leqslant 0
\end{aligned}
$$

where, by Itô's isometry,

$$
\begin{aligned}
\sum_{k=0}^{N-1} \mathbb{E}^{\prime}\left\|\int_{t_{k}}^{t_{k+1}} \Phi_{N} \mathrm{~d} W_{N}(t)\right\|_{2}^{2} & =\sum_{k=0}^{N-1} \mathbb{E}^{\prime} \int_{t_{k}}^{t_{k+1}}\left\|\Phi_{N}\right\|_{H S\left(L^{2}(D)\right)}^{2} \mathrm{~d} t \\
& =\mathbb{E}^{\prime} \int_{0}^{T}\left\|\Phi_{N}\right\|_{H S\left(L^{2}(D)\right)}^{2} \mathrm{~d} t .
\end{aligned}
$$

By equality of laws, from (2.50) and (2.51) it follows that

$$
\begin{aligned}
& \mathbb{E}^{\prime}\left[\left\|\widehat{u}_{N}(T)\right\|_{2}^{2}-\left\|u^{0}\right\|_{2}^{2}+2 \int_{0}^{T} \int_{D} a\left(x, u_{N}^{r}, \nabla u_{N}^{r}\right) \cdot \nabla u_{N}^{r} \mathrm{~d} x \mathrm{~d} t\right] \\
& \quad-\mathbb{E}^{\prime} \int_{0}^{T}\left\|\Phi_{N}\right\|_{H S\left(L^{2}(D)\right)}^{2} \mathrm{~d} t \leqslant 0 .
\end{aligned}
$$


On the other hand, Itô's formula and (2.47) yield

$$
\begin{aligned}
\left\|u_{\infty}(T)\right\|_{2}^{2}-\left\|u_{0}\right\|_{2}^{2}+2 \int_{0}^{T} \int_{D} G \cdot \nabla u_{\infty} \mathrm{d} x \mathrm{~d} t \\
=\int_{0}^{T}\left\|\Phi_{\infty}\right\|_{H S\left(L^{2}(D)\right)}^{2} \mathrm{~d} t+2 \int_{0}^{T}\left(u_{\infty}, \Phi_{\infty} \mathrm{d} W_{\infty}(t)\right)_{2},
\end{aligned}
$$

a.s. in $\Omega^{\prime}$, therefore

$$
\mathbb{E}^{\prime}\left[\left\|u_{\infty}(T)\right\|_{2}^{2}-\left\|u_{0}\right\|_{2}^{2}+2 \int_{0}^{T} \int_{D} G \cdot \nabla u_{\infty} \mathrm{d} x \mathrm{~d} t\right]-\mathbb{E}^{\prime} \int_{0}^{T}\left\|\Phi_{\infty}\right\|_{H S\left(L^{2}(D)\right)}^{2} \mathrm{~d} t=0 .
$$

From the above equation and (2.52), it follows that $\left(u^{0}=u_{0}^{\tau}\right.$ is given by lemma A.1)

$$
\begin{aligned}
\mathbb{E}^{\prime}\left\|u_{\infty}(T)\right\|_{2}^{2}+2 \mathbb{E}^{\prime} \int_{0}^{T} \int_{D} G \cdot \nabla u_{\infty} \mathrm{d} x \mathrm{~d} t-\mathbb{E}^{\prime} \int_{0}^{T}\left\|\Phi_{\infty}\right\|_{H S\left(L^{2}(D)\right)}^{2} \mathrm{~d} t \\
\geqslant \mathbb{E}^{\prime}\left\|\widehat{u}_{N}(T)\right\|_{2}^{2}+2 \mathbb{E}^{\prime} \int_{0}^{T} \int_{D} a\left(x, u_{N}^{r}, \nabla u_{N}^{r}\right) \cdot \nabla u_{N}^{r} \mathrm{~d} x \mathrm{~d} t \\
\quad-\mathbb{E}^{\prime} \int_{0}^{T}\left\|\Phi_{N}\right\|_{H S\left(L^{2}(D)\right)}^{2} \mathrm{~d} t+\left\|u_{0}\right\|_{2}^{2}-\left\|u_{0}^{\tau}\right\|_{2}^{2}
\end{aligned}
$$

hence

$$
\begin{aligned}
& 2 \mathbb{E}^{\prime} \int_{0}^{T} \int_{D} G \cdot \nabla u_{\infty} \mathrm{d} x \mathrm{~d} t \\
& \geqslant \mathbb{E}^{\prime}\left(\left\|\widehat{u}_{N}(T)\right\|_{2}^{2}-\left\|u_{\infty}(T)\right\|_{2}^{2}\right)+\mathbb{E}^{\prime} \int_{0}^{T}\left\|\Phi_{N}\right\|_{H S\left(L^{2}(D)\right)}^{2}-\left\|\Phi_{\infty}\right\|_{H S\left(L^{2}(D)\right)}^{2} \mathrm{~d} t \\
& \quad+2 \mathbb{E}^{\prime} \int_{0}^{T} \int_{D} a\left(x, u_{N}^{r}, \nabla u_{N}^{r}\right) \cdot \nabla u_{N}^{r} \mathrm{~d} x \mathrm{~d} t+\left\|u_{0}\right\|_{2}^{2}-\left\|u_{0}^{\tau}\right\|_{2}^{2}
\end{aligned}
$$

Since $\widehat{u}_{N}$ converges to $u_{\infty}$ in $L^{\ell}\left(\Omega^{\prime} ; \mathcal{C}\left([0, T] ; W^{-1, p^{\prime}}(D)\right)\right)$ for some $1 \leqslant \ell<2$, for any $t, \widehat{u}_{N}(t)$ converges to $u_{\infty}(t)$ in $L^{\ell}\left(\Omega^{\prime} ; W^{-1, p^{\prime}}(D)\right)$. On the other hand, the sequence $\widehat{u}_{N}(t)$ is bounded in $L^{2}\left(\Omega^{\prime} \times D\right)$ and one is able to conclude that $\widehat{u}_{N}(t)$ converges weakly to $u_{\infty}(t)$ in $L^{2}\left(\Omega^{\prime} \times D\right)$. Therefore, by lower semi-continuity, $\liminf \operatorname{inf\infty }_{N} \mathbb{E}^{\prime}\left\|\widehat{u}_{N}(T)\right\|_{2}^{2} \geqslant \mathbb{E}^{\prime}\left\|u_{\infty}(T)\right\|_{2}^{2}$ and since

$$
\mathbb{E}^{\prime} \int_{0}^{T}\left\|\Phi_{N}\right\|_{H S\left(L^{2}(D)\right)}^{2} \mathrm{~d} t \rightarrow \mathbb{E}^{\prime} \int_{0}^{T}\left\|\Phi_{\infty}\right\|_{H S\left(L^{2}(D)\right)}^{2} \mathrm{~d} t
$$

for $N \rightarrow \infty$, it follows that

$$
\begin{aligned}
\mathbb{E}^{\prime} \int_{0}^{T} \int_{D} G \cdot \nabla u_{\infty} \mathrm{d} x \mathrm{~d} t \geqslant & \limsup _{N \rightarrow \infty} \mathbb{E}^{\prime} \int_{0}^{T} \int_{D} a\left(x, u_{N}^{r}, \nabla u_{N}^{r}\right) \cdot \nabla u_{N}^{r} \mathrm{~d} x \mathrm{~d} t \\
& +\liminf _{N \rightarrow \infty} \mathbb{E}^{\prime}\left\|\widehat{u}_{N}(T)\right\|_{2}^{2}-\mathbb{E}^{\prime}\left\|u_{\infty}(T)\right\|_{2}^{2} \\
\geqslant & \limsup _{N \rightarrow \infty} \mathbb{E}^{\prime} \int_{0}^{T} \int_{D} a\left(x, u_{N}^{r}, \nabla u_{N}^{r}\right) \cdot \nabla u_{N}^{r} \mathrm{~d} x \mathrm{~d} t .
\end{aligned}
$$


The next step is to prove that

$$
\mathbb{E}^{\prime} \int_{0}^{T} \int_{D} G \cdot \nabla u_{\infty} \mathrm{d} x \mathrm{~d} t \leqslant \liminf _{N \rightarrow \infty} \mathbb{E}^{\prime} \int_{0}^{T} \int_{D} a\left(x, u_{N}^{r}, \nabla u_{N}^{r}\right) \cdot \nabla u_{N}^{r} \mathrm{~d} x \mathrm{~d} t
$$

Following the proof of $\left[\mathbf{1 2}\right.$, lemma 8.8, p. 208], for any $N \in \mathbb{N}^{*}$ and almost every $(\omega, t) \in \Omega^{\prime} \times(0, T)$, we define

$$
\xi_{N}(\omega, t):=\int_{D} a\left(x, u_{N}^{r}, \nabla u_{N}^{r}\right) \cdot \nabla\left(u_{N}^{r}-u_{\infty}\right) \mathrm{d} x
$$

and observe that by $(A 2)$ and Young's inequality, there exist $\delta, C>0$ such that

$$
\xi_{N}(\omega, t) \geqslant \delta \int_{D}\left|\nabla u_{N}^{r}\right|^{p} \mathrm{~d} x-C\left(\int_{D}\left|\nabla u_{\infty}\right|^{p} \mathrm{~d} x+1\right)
$$

for any $N \in \mathbb{N}^{*}$, a.s. in $\Omega^{\prime} \times(0, T)$.

As a direct consequence of (2.59), we get that $\liminf _{N \rightarrow \infty} \xi_{N}(\omega, t)>-\infty$ a.s. in $\Omega^{\prime} \times(0, T)$. If we can show that $\liminf _{N \rightarrow \infty} \xi_{N}(\omega, t) \geqslant 0$, a.s. in $\Omega^{\prime} \times$ $(0, T)$, then (2.58) would follow from Fatou's lemma. Assume that for a given $(\omega, t), \liminf _{N \rightarrow \infty} \xi_{N}(\omega, t)=a<0$. This yields the existence of a subsequence $N^{\prime}$ (depending on $\omega$ and $t$ ) such that $\lim _{N^{\prime} \rightarrow \infty} \xi_{N^{\prime}}(\omega, t)=a<0$, thus $\xi_{N^{\prime}}(\omega, t)<$ $a / 2<0$ after a certain time, considered in the next few lines as the starting point of the sequence. Then, (2.59) ensures the boundedness of $u_{N^{\prime}}^{r}(\omega, t)$ in $W_{0}^{1, p}(D)$. Since $u_{N^{\prime}}^{r}(\omega, t)$ converges to $u_{\infty}(\omega, t)$ in $L^{2}(D)$, one is able to conclude that $u_{N^{\prime}}^{r}(\omega, t)$ converges also weakly in $W_{0}^{1, p}(D)$. Since the nonlinear operator

$$
\mathcal{A}: W_{0}^{1, p}(D) \rightarrow W^{-1, p^{\prime}}(D), \quad u \mapsto \mathcal{A}(u):=-\operatorname{div} a(x, u, \nabla u)
$$

is pseudomonotone, $a=\liminf _{N^{\prime} \rightarrow \infty} \xi_{N^{\prime}}(\omega, t) \geqslant 0$. This is a contradiction and one concludes that $\liminf _{N \rightarrow \infty} \xi_{N}(\omega, t) \geqslant 0$, for almost any $(\omega, t)$ in $\Omega^{\prime} \times(0, T)$ and therefore (2.58) is true. As a consequence of (2.57) and (2.58),

$$
\lim _{N \rightarrow \infty} \mathbb{E}^{\prime} \int_{0}^{T} \int_{D} a\left(x, u_{N}^{r}, \nabla u_{N}^{r}\right) \cdot \nabla u_{N}^{r} \mathrm{~d} x \mathrm{~d} t=\mathbb{E}^{\prime} \int_{0}^{T} \int_{D} G \cdot \nabla u_{\infty} \mathrm{d} x \mathrm{~d} t
$$

and, for any $\vec{\xi} \in L^{p}\left(\Omega^{\prime} \times Q_{T}\right)^{d}$,

$$
\lim _{N \rightarrow \infty} \mathbb{E}^{\prime} \int_{0}^{T} \int_{D} a\left(x, u_{N}^{r}, \nabla u_{N}^{r}\right) \cdot\left(\nabla u_{N}^{r}-\vec{\xi}\right) \mathrm{d} x \mathrm{~d} t=\mathbb{E}^{\prime} \int_{0}^{T} \int_{D} G \cdot\left(\nabla u_{\infty}-\vec{\xi}\right) \mathrm{d} x \mathrm{~d} t .
$$

Since for almost any $(\omega, t)$ in $\Omega^{\prime} \times(0, T)$, $\liminf _{N \rightarrow \infty} \xi_{N}(\omega, t) \geqslant 0$, one gets that $\lim _{N \rightarrow \infty} \xi_{N}^{-}(\omega, t)=0$ a.s. in $\Omega^{\prime}$, where $-r^{-}:=\min (0, r)$ for $r \in \mathbb{R}$. As by $(2.59)$,

$$
0 \geqslant-\xi_{N}^{-} \geqslant-C\left(\int_{D}\left|\nabla u_{\infty}\right|^{p} \mathrm{~d} x+1\right) \in L^{1}\left(\Omega^{\prime} \times(0, T)\right),
$$

one concludes that $\xi_{N}^{-}$converges to 0 in $L^{1}\left(\Omega^{\prime} \times(0, T)\right)$. From $(2.61)$ in particular we get $\lim _{N \rightarrow \infty} \mathbb{E}^{\prime} \int_{0}^{T} \xi_{N} \mathrm{~d} t=0$, and therefore $\left(\xi_{N}\right)$ converges to 0 in $L^{1}\left(\Omega^{\prime} \times(0, T)\right)$. 
Up to a subsequence denoted by $N^{\prime}$, one can consider that $\lim _{N^{\prime} \rightarrow \infty} \xi_{N^{\prime}}=0$ a.s. in $\Omega^{\prime} \times(0, T)$ and that there exists $\Theta \in L^{1}\left(\Omega^{\prime} \times(0, T)\right)$ such that $\left|\xi_{N^{\prime}}\right| \leqslant \Theta$ for all $N^{\prime} \in \mathbb{N}^{*}$ a.e. in $\Omega^{\prime} \times(0, T)$.

Since $u_{N}^{r} \rightarrow u_{\infty}$ for $N \rightarrow \infty$ in $L^{2}(D)$ a.e. in $\Omega^{\prime} \times(0, T), \quad(2.59)$ with the above majoration by $\Theta$ yield the boundedness of $u_{N^{\prime}}^{r}(\omega, t)$ in $W_{0}^{1, p}(D)$, thus $u_{N^{\prime}}^{r}(\omega, t) \rightarrow u_{\infty}(\omega, t)$ in $W_{0}^{1, p}(D)$ and $u_{N^{\prime}}^{r}(\omega, t) \rightarrow u_{\infty}(\omega, t)$ in $L^{p}(D)$ for almost every $(\omega, t) \in \Omega^{\prime} \times(0, T)$. By assumption $(A 2)$, for any $\vec{\xi} \in L^{p}\left(\Omega^{\prime} \times Q_{T}\right)^{d}$, the properties of Nemytskii's operators yield $a\left(x, u_{N^{\prime}}^{r}(\omega, t), \vec{\xi}\right) \rightarrow a\left(x, u_{\infty}(\omega, t), \vec{\xi}\right)$ in $L^{p^{\prime}}(D)$ for almost every $(\omega, t) \in \Omega^{\prime} \times(0, T)$ and for $N^{\prime} \rightarrow \infty$, so that

$$
\lim _{N^{\prime} \rightarrow \infty} \int_{D} a\left(x, u_{N^{\prime}}^{r}, \vec{\xi}\right) \cdot\left(\nabla u_{N^{\prime}}^{r}-\vec{\xi}\right) \mathrm{d} x=\int_{D} a\left(x, u_{\infty}, \vec{\xi}\right) \cdot\left(\nabla u_{\infty}-\vec{\xi}\right) \mathrm{d} x
$$

a.e. in $\Omega^{\prime} \times(0, T)$. The same assumption ensures, for almost every $(\omega, t) \in \Omega^{\prime} \times$ $(0, T)$, the boundedness of the sequence $\left(a\left(x, u_{N^{\prime}}^{r}(\omega, t), \nabla u_{N^{\prime}}^{r}(\omega, t)\right)\right)$ in $L^{p^{\prime}}(D)$ and, up to a subsequence denoted $N^{\prime \prime}$ (depending a priori on $(\omega, t)$ ), the weak convergence of $a\left(x, u_{N^{\prime \prime}}^{r}(\omega, t), \nabla u_{N^{\prime \prime}}^{r}(\omega, t)\right)$ to some $\theta(\omega, t)$ in $L^{p^{\prime}}(D)$. By assumption $(A 1)$

$$
0 \leqslant \int_{D}\left(a\left(x, u_{N^{\prime \prime}}^{r}, \nabla u_{N^{\prime \prime}}^{r}\right)-a\left(x, u_{N^{\prime \prime}}^{r}, \vec{\xi}\right)\right) \cdot\left(\nabla u_{N^{\prime \prime}}^{r}-\vec{\xi}\right) \mathrm{d} x .
$$

Then, thanks to the above convergence, (2.62) and $\lim _{N^{\prime} \rightarrow \infty} \xi_{N^{\prime}}=0$, one gets that $0 \leqslant \int_{D}\left(\theta-a\left(x, u_{\infty}, \vec{\xi}\right)\right) \cdot\left(\nabla u_{\infty}-\vec{\xi}\right) \mathrm{d} x$ and Minty's argument yields

$$
\theta(\omega, t)=a\left(x, u_{\infty}(\omega, t), \nabla u_{\infty}(\omega, t)\right) .
$$

This implies $a\left(x, u_{N^{\prime}}^{r}(\omega, t), \nabla u_{N^{\prime}}^{r}(\omega, t)\right) \rightarrow a\left(x, u_{\infty}(\omega, t), \nabla u_{\infty}(\omega, t)\right)$ weakly in $L^{p^{\prime}}(D)$, where $N^{\prime}$ can be chosen independently of $(\omega, t) \in \Omega^{\prime} \times(0, T)$. Since $\lim _{N^{\prime} \rightarrow \infty} \xi_{N^{\prime}}(\omega, t)=0$, from (2.63) it follows that for any given $\vec{\xi} \in L^{p}\left(\Omega \times Q_{T}\right)^{d}$, for almost every $(\omega, t) \in \Omega^{\prime} \times(0, T)$,

$$
\begin{gathered}
\lim _{N^{\prime} \rightarrow \infty} \int_{D} a\left(x, u_{N^{\prime}}^{r}(\omega, t), \nabla u_{N^{\prime}}^{r}(\omega, t)\right) \cdot\left(\nabla u_{N^{\prime}}^{r}(\omega, t)-\vec{\xi}(\omega, t)\right) \mathrm{d} x \\
\quad=\int_{D} a\left(x, u_{\infty}(\omega, t), \nabla u_{\infty}(\omega, t)\right) \cdot\left(\nabla u_{\infty}(\omega, t)-\vec{\xi}(\omega, t)\right) \mathrm{d} x .
\end{gathered}
$$

Similarly to $(2.59)$, for $\vec{\xi} \in L^{p}\left(\Omega \times Q_{T}\right)^{d}$,

$$
\int_{D} a\left(x, u_{N}^{r}, \nabla u_{N}^{r}\right) \cdot\left(\nabla u_{N}^{r}-\vec{\xi}\right) \mathrm{d} x \geqslant-C\left(\int_{D}|\vec{\xi}|^{p} \mathrm{~d} x+1\right)
$$

a.e. in $\Omega^{\prime} \times(0, T)$, for all $N \in \mathbb{N}^{*}$ and, by Fatou's lemma, from (2.64) and (2.65) it follows that

$$
\begin{gathered}
\liminf _{N^{\prime} \rightarrow \infty} \mathbb{E}^{\prime} \int_{0}^{T} \int_{D} a\left(x, u_{N^{\prime}}^{r}, \nabla u_{N^{\prime}}^{r}\right) \cdot\left(\nabla u_{N^{\prime}}^{r}-\vec{\xi}\right) \mathrm{d} x \mathrm{~d} t \\
\geqslant \mathbb{E}^{\prime} \int_{0}^{T} \int_{D} a\left(x, u_{\infty}, \nabla u_{\infty}\right) \cdot\left(\nabla u_{\infty}-\vec{\xi}\right) \mathrm{d} x \mathrm{~d} t .
\end{gathered}
$$


Back to (2.61), (2.66) yields

$$
\mathbb{E}^{\prime} \int_{0}^{T} \int_{D} G \cdot\left(\nabla u_{\infty}-\vec{\xi}\right) \mathrm{d} x \mathrm{~d} t \geqslant \mathbb{E}^{\prime} \int_{0}^{T} \int_{D} a\left(x, u_{\infty}, \nabla u_{\infty}\right) \cdot\left(\nabla u_{\infty}-\vec{\xi}\right) \mathrm{d} x \mathrm{~d} t
$$

for any $\vec{\xi} \in L^{p}\left(\Omega \times Q_{T}\right)^{d}$, and therefore it follows that $G=a\left(\cdot, u_{\infty}, \nabla u_{\infty}\right)$.

\section{Uniqueness and existence of strong solutions}

In this section, we show the existence of strong solutions adapting the argument of Gyöngy and Krylov (see [8]). First, we show a pathwise $L^{1}$-contraction principle:

Proposition 3.1. Assume that for a given stochastic basis $\left(\Omega, \mathcal{F},\left(\mathcal{F}_{t}\right), P\right)$ and for an arbitrary $\Phi \in L^{2}\left(\Omega \times(0, T) ; H S\left(L^{2}(D)\right)\right)$ there exist solutions $u_{1}$, $u_{2}$ to (1.1) with respect to the initial values $u_{01}$ and $u_{02}$ in $L^{2}(D)$ respectively. Then,

$$
\int_{D}\left|u_{1}(t)-u_{2}(t)\right| \mathrm{d} x \leqslant \int_{D}\left|u_{01}-u_{02}\right| \mathrm{d} x
$$

for all $t \in[0, T]$, a.s. in $\Omega$.

Proof. For $\delta>0$, consider $\eta_{\delta}$, the Lipschitz approximation of the sign function defined by $\eta_{\delta}(r)=\max (-1, \min (r / \delta, 1))$. Now, for $\delta>0$ and $r \in \mathbb{R}$ we define $N_{\delta}(r):=\int_{0}^{r} \eta_{\delta}(s) \mathrm{d} s$. Subtracting the equation for $u_{2}$ from the equation for $u_{1}$, the noise terms are eliminated and one gets pathwise the PDE

$$
\partial_{t}\left(u_{1}-u_{2}\right)-\operatorname{div}\left[a\left(\cdot, u_{1}, \nabla u_{1}\right)-a\left(\cdot, u_{2}, \nabla u_{2}\right)+F\left(u_{1}\right)-F\left(u_{2}\right)\right]=0 .
$$

Testing the equation with $\eta_{\delta}\left(u_{1}(t)-u_{2}(t)\right)$, we get $I_{1}=I_{2}+I_{3}$ for all $t \in[0, T]$ a.s. in $\Omega$, where

$$
\begin{aligned}
& I_{1}=\int_{D} N_{\delta}\left(u_{1}-u_{2}\right)(t) \mathrm{d} x-\int_{D} N_{\delta}\left(u_{01}-u_{02}\right) \mathrm{d} x \\
& I_{2}=-\int_{0}^{t} \int_{D}\left(a\left(x, u_{1}, \nabla u_{1}\right)-a\left(x, u_{2}, \nabla u_{2}\right) \cdot \nabla\left(u_{1}-u_{2}\right) \eta_{\delta}^{\prime}\left(u_{1}-u_{2}\right) \mathrm{d} x \mathrm{~d} s\right. \\
& I_{3}=-\int_{0}^{t} \int_{D}\left(F\left(u_{1}\right)-F\left(u_{2}\right)\right) \cdot \nabla\left(u_{1}-u_{2}\right) \eta_{\delta}^{\prime}\left(u_{1}-u_{2}\right) \mathrm{d} x \mathrm{~d} s
\end{aligned}
$$

and $\eta_{\delta}^{\prime}\left(u_{1}-u_{2}\right)=1 / \delta \chi_{\left\{\left|u_{1}-u_{2}\right|<\delta\right\}} \geqslant 0$ for any $\delta>0$. Now we write $I_{2}=I_{2,1}+I_{2,2}$ where, by $(A 1)$

$$
I_{2,1}=-\int_{0}^{t} \int_{D}\left(a\left(x, u_{1}, \nabla u_{1}\right)-a\left(x, u_{1}, \nabla u_{2}\right)\right) \cdot \nabla\left(u_{1}-u_{2}\right) \eta_{\delta}^{\prime}\left(u_{1}-u_{2}\right) \mathrm{d} x \mathrm{~d} s \leqslant 0
$$


Using (A3) and Hölder's inequality we get

$$
\begin{aligned}
\left|I_{2,2}\right| & =\left|\int_{0}^{t} \int_{D}\left(a\left(x, u_{1}, \nabla u_{2}\right)-a\left(x, u_{2}, \nabla u_{2}\right)\right) \cdot \nabla\left(u_{1}-u_{2}\right) \eta_{\delta}^{\prime}\left(u_{1}-u_{2}\right) \mathrm{d} x \mathrm{~d} s\right| \\
& \leqslant \int_{0}^{t} \int_{D}\left(C_{a}^{4}\left|\nabla u_{2}\right|^{p-1}+h(x)\right)\left|u_{1}-u_{2}\right|\left|\nabla\left(u_{1}-u_{2}\right)\right| \eta_{\delta}^{\prime}\left(u_{1}-u_{2}\right) \mathrm{d} x \mathrm{~d} s \\
& \leqslant C\left(\int_{\left\{\left|u_{1}-u_{2}\right|\right\}<\delta}\left|\nabla\left(u_{1}-u_{2}\right)\right|^{p} \mathrm{~d} x \mathrm{~d} s\right)^{1 / p},
\end{aligned}
$$

where $C \geqslant 0$ is a constant not depending on $\delta>0$. Therefore, $\lim _{\delta \rightarrow 0^{+}} I_{2,2}=0$ and consequently it follows that $\limsup _{\delta \rightarrow 0^{+}} I_{2} \leqslant 0$ for all $t \in[0, T]$, a.s. in $\Omega$. Since $N_{\delta}\left(u_{1}-u_{2}\right)(t)$ converges to $\left|\left(u_{1}-u_{2}\right)(t)\right|$ for $\delta \rightarrow 0^{+}$, and $\left|N_{\delta}\left(u_{1}-u_{2}\right)(t)\right| \leqslant$ $\left|\left(u_{1}-u_{2}\right)(t)\right|$ for all $\delta>0$, it follows that

$$
\lim _{\delta \rightarrow 0^{+}} I_{1}=\int_{D}\left|u_{1}(t)-u_{2}(t)\right| \mathrm{d} x-\int_{D}\left|u_{01}-u_{02}\right| \mathrm{d} x
$$

for any $t \in[0, T]$ a.s. in $\Omega$. For $L \geqslant 0$ being the Lipschitz constant of $F$ we have

$$
\begin{aligned}
\left|I_{3}\right| & \leqslant \frac{1}{\delta} \int_{\left\{\left|u_{1}-u_{2}\right|<\delta\right\}}\left|F\left(u_{1}\right)-F\left(u_{2}\right)\right|\left|\nabla\left(u_{1}-u_{2}\right)\right| \mathrm{d} x \mathrm{~d} s \\
& \leqslant \frac{L}{\delta} \int_{\left\{\left|u_{1}-u_{2}\right|<\delta\right\}}\left|u_{1}-u_{2}\right|\left|\nabla\left(u_{1}-u_{2}\right)\right| \mathrm{d} x \mathrm{~d} s \\
& \leqslant L \int_{\left\{\left|u_{1}-u_{2}\right|<\delta\right\}}\left|\nabla\left(u_{1}-u_{2}\right)\right| \mathrm{d} x \mathrm{~d} s,
\end{aligned}
$$

and it follows, by arguments similar to the ones on p. 6 , that $\lim _{\delta \rightarrow 0^{+}} I_{3}=$ $\int_{\left\{u_{1}=u_{2}\right\}}\left|\nabla\left(u_{1}-u_{2}\right)\right| \mathrm{d} x \mathrm{~d} s=0$ a.s. in $\Omega$ and the result holds true.

In particular, proposition 3.1 implies that whenever a solution (in the sense of definition 1.1) to (1.1) exists, it is unique. The following lemma (see, e.g. [8, lemma 1.1 , pp. 144-145]) contains a suitable necessary and sufficient condition for strong convergence:

Lemma 3.2. Let $V$ be a Polish space equipped with the Borel $\sigma$-algebra. A sequence of $V$-valued random variables $\left(X_{n}\right)$ converges in probability if and only if for every pair of subsequences $\left(X_{l}\right)$ and $\left(X_{m}\right)$ there exists a joint subsequence $\left(X_{l_{k}}, X_{m_{k}}\right)$ which converges for $k \rightarrow \infty$ in law to a probability measure $\mu$ such that $\mu(\{(w, z) \in$ $V \times V \mid w=z\})=1$.

LEMMA 3.3. The sequence of piecewise affine approximations $\left(\widehat{u}_{N}\right)$ converges in probability on $(\Omega, \mathcal{F}, P)$ and there exists a strong solution to (1.1).

Proof. We apply lemma 3.2 to prove the assertion. Let $\left(\widehat{u}_{K}\right)$, and $\left(\widehat{u}_{L}\right)$ be a pair of subsequences of $\left(\widehat{u}_{N}\right)$. Then, repeating the arguments of $\S 2.3$, it follows that the 
random vector $\left(Y_{K, L}\right)$ defined by

$$
\left(u_{K}^{r}-\widehat{u}_{K}, \widehat{u}_{K}, \widehat{u}_{K}-\widehat{M}_{K}, M_{K}, \widehat{M}_{K}, \Phi_{K}, u_{L}^{r}-\widehat{u}_{L}, \widehat{u}_{L}, \widehat{u}_{L}-\widehat{M}_{L}, M_{L}, \widehat{M}_{L}, \Phi_{L}, W\right)
$$

is tight on the appropriate product space, and therefore relatively compact; thus we can extract a joint subsequence $\left(Y_{K_{j}, L_{j}}\right)$ which converges in law. Thus, in particular, there exist probability measures $\mu_{1}, \mu_{2}$ such that $\left(\mu_{1}, \mu_{2}\right)=\lim _{j \rightarrow \infty} \mathcal{L}\left(\widehat{u}_{K_{j}}, \widehat{u}_{L_{j}}\right)$. Now we continue as in $\S \S 2.3,2.4$ and 2.5: passing to a new probability space $\left(\Omega^{\prime}, \mathcal{F}^{\prime}, P^{\prime}\right)$ and not changing notation for random variables with the same law, we find random variables $u_{\infty}^{1}, u_{\infty}^{2}$ such that $\mathcal{L}\left(u_{\infty}^{1}\right)=\mu_{1}, \mathcal{L}\left(u_{\infty}^{2}\right)=\mu_{2}$ and, since we have the strong convergences of $\widehat{M}_{N}, M_{N}$ and $\Phi_{N}$, it implies that $\widehat{M}_{K_{j}}, M_{K_{j}}, \widehat{M}_{L_{j}}, M_{L_{j}}$ and $\Phi_{K_{j}}, \Phi_{L_{j}}$ are converging to the same limits and

$$
u_{\infty}^{i}(t)-u_{0}-\int_{0}^{t} \operatorname{div}\left(a\left(x, u_{\infty}^{i}, \nabla u_{\infty}^{i}\right)+F\left(u_{\infty}^{i}\right)\right) \mathrm{d} s=\int_{0}^{t} \Phi_{\infty} \mathrm{d} W_{\infty}(s)
$$

for $i=1,2$, where $W_{\infty}$ is a cylindrical $\left(\mathcal{F}_{t}^{\infty}\right)$-Wiener process and $\left(\mathcal{F}_{t}^{\infty}\right)$ is the augmentation of the filtration $\sigma\left(\Phi(s), W_{\infty}(s), u_{\infty}^{1}(s), u_{\infty}^{2}(s)\right)_{0 \leqslant s \leqslant t}, t \in[0, T]$. Now, from proposition 3.1 it follows that $u_{\infty}^{1}=u_{\infty}^{2}$ in $L^{2}\left(\Omega^{\prime} ; \mathcal{C}\left([0, T] L^{2}(D)\right)\right)$ and for the joint law $\left(\mu_{1}, \mu_{2}\right)$ on $\mathcal{C}\left([0, T] ; W^{-1, p^{\prime}}(D)\right)^{2}$ it follows that

$$
\left(\mu_{1}, \mu_{2}\right)\left(\left\{(w, z) \in \mathcal{C}\left([0, T] ; W^{-1, p^{\prime}}(D)\right) \times \mathcal{C}\left([0, T] ; W^{-1, p^{\prime}}(D)\right) \mid w=z\right\}\right)=1
$$

and the convergence in probability of the sequence $\left(\widehat{u}_{N}\right)$ follows from lemma 3.2. Again, since we have the strong convergence of $\widehat{M}_{N}, M_{N}$ and $\Phi_{N}$ in $\Omega$, it is possible to recover the steps of the proof of existence of a solution in $\Omega$ and therefore a strong solution exists.

\section{Appendix A. On the regularization of the initial condition}

Lemma A.1. For any positive $\tau$, there exists $u_{0}^{\tau} \in W_{0}^{1, p}(D)$ such that $\left(u_{0}^{\tau}\right)$ converges to $u_{0}$ in $L^{2}(D)$ for $\tau \rightarrow 0^{+}$and satisfies $\frac{1}{2}\left\|u_{0}^{\tau}\right\|_{L^{2}(D)}^{2}+\tau\left\|\nabla u_{0}^{\tau}\right\|_{p}^{p} \leqslant \frac{1}{2}\left\|u_{0}\right\|_{L^{2}(D)}^{2}$.

Proof. Denote by $u^{\tau}$ the unique solution in $W_{0}^{1, p}(D)$ to the problem $u^{\tau}-\tau \Delta_{p} u^{\tau}=$ $u_{0}$. Using the solution $u^{\tau}$ as a test function in the equation, one gets

$$
\left\|u^{\tau}\right\|_{L^{2}(D)}^{2}+\tau\left\|\nabla u^{\tau}\right\|_{p}^{p}=\int_{D} u_{0} u^{\tau} \mathrm{d} x \leqslant \frac{1}{2}\left\|u_{0}\right\|_{L^{2}(D)}^{2}+\frac{1}{2}\left\|u^{\tau}\right\|_{L^{2}(D)}^{2},
$$

and consequently, up to a not relabelled subsequence, $\left(u^{\tau}\right)$ converges weakly in $L^{2}(D)$ to a given $v$ with the additional information that $\left\|u^{\tau}\right\|_{L^{2}(D)} \leqslant\left\|u_{0}\right\|_{L^{2}(D)}$. Therefore, for any $\varphi \in \mathcal{D}(D)$, one has

$$
\int_{D} u_{0} \varphi \mathrm{d} x=\int_{D} u^{\tau} \varphi \mathrm{d} x+\tau \int_{D}\left|\nabla u^{\tau}\right|^{p-2} \nabla u^{\tau} \nabla \varphi \mathrm{d} x \rightarrow \int_{D} v \varphi \mathrm{d} x
$$

and therefore $\left(u^{\tau}\right)$ converges to $v=u_{0}$ in the sense of distributions and weakly in $L^{2}(D)$. As a consequence, the whole sequence $\left(u^{\tau}\right)$ converges to $u_{0}$ weakly in $L^{2}(D)$ and strongly in $L^{2}(D)$ thanks to the uniform convexity property and the above inequality. Therefore, $u_{0}^{\tau}:=u^{\tau}$ for all $\tau>0$. 


\section{A.1. The lemma of Brezis-Lieb}

Lemma A.2. Let $(\Omega, \mathcal{F}, P)$ be a probability space $\left(u_{n}\right) \subset L^{2}(\Omega ; X)$ where $X$ is a separable Banach space such that $u_{n} \rightarrow u$ in $X$ a.s. in $\Omega$ for some $u \in L^{2}(\Omega ; X)$ and $\sup _{n} \mathbb{E}\left\|u_{n}\right\|_{X}^{2}=: M<+\infty$. Then,

$$
\lim _{n \rightarrow \infty} \mathbb{E}\left(\left\|u_{n}\right\|_{X}^{2}-\left\|u_{n}-u\right\|_{X}^{2}\right)=\mathbb{E}\|u\|_{X}^{2}
$$

In particular, without using any argument of uniform convexity, a.s. convergence of $\left(u_{n}\right)$ to $u$ in $X$ and $\lim _{n \rightarrow \infty} \mathbb{E}\left\|u_{n}\right\|_{X}^{2}=\mathbb{E}\|u\|_{X}^{2}$ yields the convergence of $\left(u_{n}\right)$ to $u$ in $L^{2}(\Omega ; X)$.

Proof. For $n \in \mathbb{N}^{*}$ and $\varepsilon>0$ we define $X_{n}:=\left|\left\|u_{n}\right\|_{X}^{2}-\left\|u_{n}-u\right\|_{X}^{2}-\|u\|_{X}^{2}\right|, Y_{n}^{\varepsilon}:=$ $\left(X_{n}-\varepsilon\left\|u_{n}\right\|_{X}^{2}\right)^{+}$. For all $n \in \mathbb{N}^{*}$ we have

$$
\begin{aligned}
X_{n} & \leqslant\|u\|_{X}^{2}+\left|\left\|u_{n}\right\|_{X}-\left\|u_{n}-u\right\|_{X}\right|\left(\left\|u_{n}\right\|_{X}+\left\|u_{n}-u\right\|_{X}\right) \\
& \leqslant\|u\|_{X}^{2}+\|u\|_{X}\left(\left\|u_{n}\right\|_{X}+\left\|u_{n}-u\right\|_{X}\right) \\
& \leqslant\|u\|_{X}^{2}+2\|u\|_{X}\left\|u_{n}\right\|_{X}+\|u\|_{X}^{2} \leqslant\|u\|_{X}^{2}+\frac{1}{\varepsilon}\|u\|_{X}^{2}+\varepsilon\left\|u_{n}\right\|_{X}^{2}+\|u\|_{X}^{2} \\
& =\left(2+\frac{1}{\varepsilon}\right)\|u\|_{X}^{2}+\varepsilon\left\|u_{n}\right\|_{X}^{2}
\end{aligned}
$$

and from (A.2) it follows that there exists a constant $C_{\varepsilon} \geqslant 0$ not depending on $n \in$ $\mathbb{N}^{*}$ such that $0 \leqslant Y_{n}^{\varepsilon} \leqslant C_{\varepsilon}\|u\|_{X}^{2}$ for all $n \in \mathbb{N}^{*}$, a.s. in $\Omega$. Since $\lim _{n \rightarrow \infty}\left\|u_{n}-u\right\|_{X}^{2}=$ 0 a.s. in $\Omega$, it follows that $\lim _{n \rightarrow \infty} Y_{n}^{\varepsilon}=0$. Combining these two results, we get that the convergence also holds in $L^{1}(\Omega)$. Now, $\mathbb{E}\left[X_{n}\right]=\mathbb{E}\left[X_{n}-\varepsilon\left\|u_{n}\right\|_{X}^{2}\right]+\varepsilon \mathbb{E}\left\|u_{n}\right\|_{X}^{2} \leqslant$ $\mathbb{E}\left[Y_{n}\right]+\varepsilon M$, and therefore it follows that $\limsup _{n \rightarrow \infty} \mathbb{E}\left[X_{n}\right] \leqslant \varepsilon M$, for any $\varepsilon>0$. Therefore we get $\limsup _{n \rightarrow \infty} \mathbb{E}\left[X_{n}\right] \leqslant 0$ and this yields (A.1).

\section{A.2. Some technical tools}

\section{A.2.1. Laws and subsets}

Lemma A.3. Let $(\Omega, A, P)$ and $(\widetilde{\Omega}, \tilde{A}, \tilde{P})$ be complete probability spaces, $E, F$ be separable Banach spaces with a continuous embedding of $F$ in $E$ and consider $X$ : $\Omega \rightarrow E$ and $\widetilde{X}: \widetilde{\Omega} \rightarrow E$, two random variables with the same law on $E$.

If $X$ takes values in $F$, then it is a random variable with values in $F$. Moreover, $\widetilde{X}$ is also a random variable with values in $F$, with the same law on $F$.

Proof. Assume that $X(\Omega) \subset F$. By [16, theorem 1.1, p. 5], $\mathcal{B}(F) \subset \mathcal{B}(E)$ and $X$ : $\Omega \rightarrow F$ is a random variable. Moreover, $F \in \mathcal{B}(E)$ and $0=P[X \notin F]=\widetilde{P}[\widetilde{X} \notin F]$ thus it follows that $\widetilde{X}(\widetilde{\Omega}) \subset F$. The same argument yields that $\widetilde{X}: \widetilde{\Omega} \rightarrow F$ is a random variable and that $X$ and $\widetilde{X}$ have the same law on $F$, namely the restriction of their law to $F$.

A.2.2. On predictability Let $(\Omega, A, P)$ be a complete probability space, $E, F$ be separable Banach spaces, and $X: \Omega \times[0, T] \rightarrow E$ a process with continuous trajectories and denote by $\left(\mathcal{F}_{t}\right)$ its natural filtration. 
Lemma A.4. For any $t \in[0, T]$, denote by $X_{\mid[0, t]}: \Omega \rightarrow \mathcal{C}([0, t] ; E), \omega \mapsto X(\omega, \cdot)$. Then, $\sigma\left\{X_{\mid[0, t]}\right\}$, the sigma algebra generated by the random variable $X_{\mid[0, t]}$, is $\mathcal{F}_{t}$.

Proof. Since $X$ is continuous with respect to $t$ and adapted to $\left(\mathcal{F}_{t}\right)$, it is progressively measurable, thus $\forall t \in[0, T], X: \Omega \times[0, t] \rightarrow E \quad$ is $\mathcal{F}_{t} \times B(0, t)$ measurable. Therefore, $X: \Omega \times[0, t] \rightarrow E$ is a Carathéodory function on $\left(\Omega, \mathcal{F}_{t}\right) \times[0, t]$ to $E$, and thanks to $[\mathbf{1}$, theorem 1$]$, the random variable $X_{\mid[0, t]}: \Omega \rightarrow \mathcal{C}([0, t] ; E), \omega \mapsto$ $X(\omega, \cdot)$ is $\mathcal{F}_{t}$-measurable. Hence, $\sigma\left\{X_{\mid[0, t]}\right\} \subset \mathcal{F}_{t}$.

On the other hand, for any $s \in[0, t]$, the mapping $\delta_{s}: \mathcal{C}([0, t] ; E) \rightarrow E, g \mapsto g(s)$ is continuous. Thus, $\omega \mapsto X(\omega, s)=\delta_{s}\left(X_{\mid[0, t]}(\omega)\right)$ is $\sigma\left\{X_{\mid[0, t]}\right\}$ measurable for any such $s$ and $\sigma\left\{X_{\mid[0, t]}\right\}=\mathcal{F}_{t}$.

Denote by $Y: \Omega \rightarrow F$ a $\mathcal{F}_{t}$-measurable random variable. Then, $Y$ is $\sigma\left\{X_{\mid[0, t]}\right\}$ measurable and, by $[\mathbf{9}$, lemma 1.13 p. 7$],{ }^{3}$ there exists a Borel function $h$ from $\mathcal{C}([0, t], E)$ to $F$ such that $Y=h\left(X_{\mid[0, t]}\right)$.

Denote by $\psi$ the nonnegative mapping defined on $\mathcal{C}([0, T] ; E) \times F$ by $\psi(u, v)=$ $\left\|h\left[u_{\mid[0, t]}\right]-v\right\|_{F}$ where $u_{\mid[0, t]}$ denotes the restriction to $[0, t]$ of $u$. The mapping $u \mapsto u_{\mid[0, t]}$ is continuous from $\mathcal{C}([0, T] ; E)$ into $\mathcal{C}([0, t] ; E)$, and $h$ is a Borel function from $\mathcal{C}([0, t] ; E)$ into $F$. Therefore, $(u, v) \mapsto h\left[u_{\mid[0, t]}\right]-v$ is a Borel function from $\mathcal{C}([0, T] ; E) \times F$ into $F$, hence $\psi$ is a Borel function.

Consider another complete probability space $(\widetilde{\Omega}, \tilde{A}, \tilde{P})$, a random variable $\tilde{Y}$ : $\widetilde{\Omega} \rightarrow F$, and a pathwise continuous process $\widetilde{X}: \widetilde{\Omega} \times[0, T] \rightarrow E$ with its natural filtration $\left(\widetilde{\mathcal{F}}_{t}\right)$.

Lemma A.5. If $(X, Y)$ and $(\tilde{X}, \widetilde{Y})$ have the same law, $\widetilde{Y}$ is $\widetilde{\mathcal{F}}_{t}$-measurable.

Proof. Indeed, $\mathbb{E}[\psi(X, Y)]=\tilde{\mathbb{E}}[\psi(\tilde{X}, \tilde{Y})]$ and since $0=\mathbb{E}[\psi(X, Y)]$, one has also $\tilde{\mathbb{E}}[\psi(\widetilde{X}, \widetilde{Y})]=0$. Therefore, $\widetilde{Y}=h\left(\widetilde{X}_{\mid[0, t]}\right)$ and $\widetilde{Y}$ is $\sigma\left\{\widetilde{X}_{\mid[0, t]}\right\}$, thus $\widetilde{\mathcal{F}}_{t}$-measurable since what has been developed above for $X$ holds also for $\widetilde{X}$.

A similar result holds concerning continuous processes:

LEMmA A.6. If $Y: \Omega \times[0, T] \rightarrow F$ and $\widetilde{Y}: \widetilde{\Omega} \times[0, T] \rightarrow F$ are continuous processes such that $Y$ is $\left(\mathcal{F}_{t}\right)$-adapted and $(X, Y),(\tilde{X}, \widetilde{Y})$ have the same law $\mu$ on $\mathcal{C}([0, T] ; E) \times \mathcal{C}([0, T] ; F)$, then $\widetilde{Y}$ is $\left(\widetilde{\mathcal{F}}_{t}\right)$-predictable.

Proof. Indeed, $Y$ is also progressively measurable and, for any $t, Y_{\mid[0, t]}:\left(\Omega, \mathcal{F}_{t}\right) \rightarrow$ $\mathcal{C}([0, t], F), \omega \mapsto Y(\omega, \cdot)$ is measurable, thus $\sigma\left\{X_{\mid[0, t]}\right\}$ measurable. Then, there exists a Borel function $h_{t}$ such that $Y_{\mid[0, t]}=h_{t}\left(X_{\mid[0, t]}\right)$. By using the above idea, for given $(u, v) \in \mathcal{C}([0, T] ; E) \times \mathcal{C}([0, T] ; F), \psi(u, v)=\left\|h\left[u_{\mid[0, t]}\right]-v_{\mid[0, t]}\right\|_{\mathcal{C}([0, t], F)}$ is a non-negative Borel function and $0=\mathbb{E}\left[\psi\left(X_{\mid[0, t]}, Y_{\mid[0, t]}\right)\right]=\tilde{\mathbb{E}}\left[\psi\left(\widetilde{X}_{\mid[0, t]}, \widetilde{Y}_{\mid[0, t]}\right)\right]$. Thus, $\widetilde{Y}_{\mid[0, t]}$ is $\widetilde{X}_{\mid[0, t]}$ measurable, thus $\widetilde{\mathcal{F}}_{t}$ measurable, and, as a Carathéodory function, it is progressive, then predictable.

\footnotetext{
${ }^{3}[\mathbf{9}$, lemma 1.13, p. 7]: fix two measurable functions $f$ and $g$ from a measurable space $(\Omega, \mathcal{A})$ into some measurable Borel spaces $(S, \mathcal{S})$ and $(T, \mathcal{T}) . f$ is $g$-measurable (i.e. $\sigma(f) \subset \sigma(g))$ if and only if there exists some measurable mapping $h: T \rightarrow S$ with $f=h \circ g$.
} 


\section{A.3. From $\Omega$ to $\Omega^{\prime}$}

In this section, we are interested in proving that the time discretization scheme is preserved by changing the probability space. Following the notations of $\S \S 2.3 .1$ and 2.3.2, by Skorokhod representation, we consider a vector of random elements $Y_{N}^{\prime}=\left(u_{N}^{\prime r}-\widehat{u}_{N}^{\prime}, \widehat{u}_{N}^{\prime}, \widehat{u}_{N}^{\prime}-\widehat{M}_{N}^{\prime}, M_{N}^{\prime}, \widehat{M}_{N}^{\prime}, \Phi_{N}^{\prime}, W_{N}^{\prime}\right)$ with the same law $\nu_{N}$ as $Y_{N}$ on $\mathcal{X}$. Let us notice that thanks to Appendix A.2.1, $Y_{N}$ and $Y_{N}^{\prime}$ have both the same law on $\left[L^{2}\left(Q_{T}\right) \cap L^{p}\left(0, T ; W_{0}^{1, p}(D)\right)\right]^{2} \times W^{1, q, p^{\prime}}\left(0, T ; W_{0}^{1, q}(D), W^{-1, p^{\prime}}(D)\right) \times$ $\left[\mathcal{C}\left([0, T] ; L^{2}(D)\right)\right]^{2} \times L^{2}\left(0, T ; H S\left(L^{2}(D)\right)\right) \times \mathcal{C}([0, T] ; U)$ denoted $\mathcal{X}^{\prime}$ where $q=$ $\min (2, p)$. Note that the semi-implicit Euler-Maruyama scheme (2.1) means, for any $k$,

$$
\left[\widehat{u}_{N}-\widehat{M}_{N}\right]\left(t_{k+1}\right)-\left[\widehat{u}_{N}-\widehat{M}_{N}\right]\left(t_{k}\right)-\int_{t_{k}}^{t_{k+1}} \operatorname{div}\left(a\left(x, u_{N}^{r}, \nabla u_{N}^{r}\right)+F\left(u_{N}^{r}\right)\right) \mathrm{d} t=0
$$

and since for any $\varphi \in \mathcal{C}_{c}^{\infty}(D)$, the following mapping $\Psi$ defined by

$$
\begin{aligned}
\Psi\left(Y_{N}\right)= & \mid \int_{D} \varphi\left[\widehat{u}_{N}-\widehat{M}_{N}\right]\left(t_{k+1}\right)-\varphi\left[\widehat{u}_{N}-\widehat{M}_{N}\right]\left(t_{k}\right) \\
& +\int_{t_{k}}^{t_{k+1}}\left(a\left(x, u_{N}^{r}, \nabla u_{N}^{r}\right)+F\left(u_{N}^{r}\right)\right) \nabla \varphi \mathrm{d} t \mathrm{~d} x \mid
\end{aligned}
$$

is a positive Borel function on $\mathcal{X}^{\prime}$, one gets that

$$
\left[\widehat{u}_{N}^{\prime}-\widehat{M}_{N}^{\prime}\right]\left(t_{k+1}\right)-\left[\widehat{u}_{N}^{\prime}-\widehat{M}_{N}^{\prime}\right]\left(t_{k}\right)-\int_{t_{k}}^{t_{k+1}} \operatorname{div}\left(a\left(x, u_{N}^{\prime}, \nabla u_{N}^{\prime}\right)+F\left(u_{N}^{\prime r}\right)\right) \mathrm{d} t=0 .
$$

For a fixed $N \in \mathbb{N}^{*}, \quad u_{N}^{r}$ is a $W_{0}^{1, p}(D)$-valued, right continuous step function on the fixed time discretization $\left(t_{k}\right)_{k=0}^{N}$ : it can be identified with an element of $W_{0}^{1, p}(D)^{N}$ by the Borel measurable mapping $\sum_{k=1}^{N} u_{k} 1_{\left[t_{k-1}, t_{k}\right)} \mapsto$ $\left(u_{1}, \ldots, u_{N}\right)$. By Appendix A.2.1, one concludes that $u_{N}^{\prime}$ follows the same structure: $\sum_{k=1}^{N} u_{k}^{\prime} 1_{\left[t_{k-1}, t_{k}\right)}$ and

$$
\int_{t_{k}}^{t_{k+1}} \operatorname{div}\left(a\left(x, u_{N}^{\prime}, \nabla u_{N}^{\prime r}\right)+F\left(u_{N}^{\prime}\right)\right) \mathrm{d} t=\tau \operatorname{div}\left(a\left(x, u_{k+1}^{\prime}, \nabla u_{k+1}^{\prime}\right)+F\left(u_{k+1}^{\prime}\right)\right) .
$$

By a similar reasoning, $\Phi_{N}^{\prime}, \widehat{u}_{N}^{\prime}$ and $\widehat{M}_{N}^{\prime}$ are respectively right continuous step function and piecewise affine continuous functions for the two last ones. Taking into account those information, one gets that the discretization is conserved by changing the probability space, i.e.

$$
u^{\prime k+1}-u^{\prime k}-\tau \operatorname{div}\left(a\left(x, u^{\prime k+1}, \nabla u^{\prime k+1}\right)+F\left(u^{\prime k+1}\right)\right)=\Phi_{k}^{\prime} \Delta_{k+1} W_{N} .
$$

Then, by Appendix A.2.2, since $\Phi_{k}$ is $\mathcal{F}_{t_{k}}$ measurable where $\left(\mathcal{F}_{t}\right)$ is the filtration generated by $W$, then, $\Phi_{k}^{\prime}$ will be $\mathcal{F}_{t_{k}}^{\prime}$ measurable where $\left(\mathcal{F}_{t}^{\prime}\right)$ is the filtration generated by $W_{N}$. Therefore, the time discretization (2.1) is totally recovered and by the uniqueness of lemma 2.1, $u_{k}^{\prime}$ is $\mathcal{F}_{t_{k}}^{\prime}$ measurable for any $k$. 
Remark A.1. Note that, as a by-product, one gets that

$$
\begin{aligned}
\mathbb{E}^{\prime}\left\|\Phi_{N}^{\prime}\right\|_{L^{2}\left(0, T ; H S\left(L^{2}(D)\right)\right)}^{2} & =\mathbb{E}\left\|\Phi_{N}\right\|_{L^{2}\left(0, T, H S\left(L^{2}(D)\right)\right)}^{2} \rightarrow \mathbb{E}\|\Phi\|_{L^{2}\left(0, T ; H S\left(L^{2}(D)\right)\right)}^{2}, \\
\mathbb{E}^{\prime}\left\|\widehat{M}_{N}^{\prime}\right\|_{\mathcal{C}\left([0, T] ; L^{2}(D)\right)}^{2} & =\mathbb{E}\left\|\widehat{M}_{N}\right\|_{\mathcal{C}\left([0, T] ; L^{2}(D)\right)}^{2} \rightarrow \mathbb{E}\left\|\int_{0} \Phi d W(t)\right\|_{\mathcal{C}\left([0, T] ; L^{2}(D)\right)}^{2} \Phi
\end{aligned}
$$

for $N \rightarrow \infty$,

$$
\begin{gathered}
\mathbb{E}^{\prime} \sup _{t \in[0, T]}\left\|\Phi_{N}^{\prime}(t)\right\|_{H S\left(L^{2}(D)\right)}^{2}=\mathbb{E} \sup _{t \in[0, T]}\left\|\Phi_{N}(t)\right\|_{H S\left(L^{2}(D)\right)} \leqslant \mathbb{E}\|\Phi\|_{\mathcal{C}\left([0, T] ; H S\left(L^{2}(D)\right)\right)}^{2}, \\
\mathbb{E}^{\prime}\left\|W_{N}\right\|_{\mathcal{C}([0, T] ; U)}^{2}=\mathbb{E}\|W\|_{\mathcal{C}([0, T] ; U)}^{2}
\end{gathered}
$$

for all $N \in \mathbb{N}^{*}$ and all the a priori estimates developed in lemmas 2.2 to 2.6 remain true on $\left(\Omega^{\prime}, \mathcal{F}^{\prime}, P^{\prime}\right)$.

\section{References}

1 J. Appell and M. Váth, The space of Carathéodory functions. In Nonlinear analysis and related problems (Russian), vol. 2 of Tr. Inst. Mat. (Minsk), pp. 39-43. Natl. Akad. Nauk Belarusi, Inst. Mat. (Minsk, 1999).

2 P. Billingsley. Convergence of probability measures (New York: Wiley, 1999).

3 G. Da Prato and J. Zabczyk, Stochastic equations in infinite dimensions. Vol. 152 of Encyclopedia of Mathematics and its Applications, 2nd edn (Cambridge: Cambridge University Press, 2014).

4 A. Debussche, N. Glatt-Holtz and R. Temam. Local martingale and pathwise solutions for an abstract fluids model. Physica D 240 (2011), 1123-1144.

5 R. E. Edwards. Functional analysis (New York: Dover Publications Inc., 1995).

6 F. Flandoli and D. Gatarek. Martingale and stationary solutions for stochastic NavierStokes equations. Probab. Theory Relat. Fields 102 (1995), 367-391.

7 A. Garsia, E. Rodemich and H. Rumsey. A real variable lemma and the continuity of paths of some Gaussian processes. Indiana Univ. Math. J. 20 (1970/71), 565-578.

8 I. Gyöngy and N. Krylov. Existence of strong solutions for Itô's stochastic equations via approximations. Probab. Theory Relat. Fields 105 (1996), 143-158.

9 O. Kallenberg. Foundations of modern probability (New York: Springer, 2002).

10 W. Liu and M. Róckner. Stochastic partial differential equations: an introduction (Cham: Springer, 2015).

11 E. Pardoux, Équations aux dérivées partielles stochastiques non linéaires monotones, Ph.D. Thesis, University of Paris Sud, 1975.

12 T. Roubíček. Nonlinear partial differential equations with applications (Basel: Springer, 2013).

13 J. Simon. Compact sets in the space $L^{p}(0, T ; B)$. Ann. Mat. Pura Appl. 146 (1987), 65-96.

14 D. W. Stroock and S. R. S. Varadhan. Multidimensional diffusion processes (Berlin/New York: Springer, 1979).

15 R. Temam. Navier-Stokes equations. Theory and numerical analysis (Amsterdam/New York/Oxford: North-Holland Publishing Co., 1977).

16 N. N. Vakhania, V. I. Tarieladze and S. A. Chobanyan. Probability distributions on Banach spaces (Dordrecht/Boston: D. Reidel Publishing Company, 1987).

17 G. Vallet and A. Zimmermann. Well-posedness for a pseudomonotone evolution problem with multiplicative noise. J. Evol. Equ. 19 (2019), 153-202.

18 K. Yosida, Functional analysis. Classics in Mathematics, vol. 123 (Berlin/Heidelberg: Springer, 1995). 